\title{
Which findings should be published?
}

\section{Citation}

Frankel, Alexander, and Maximilian Kasy. 2018. "Which findings should be published?" Working paper, Department of Economics, Harvard University.

\section{Permanent link}

http://nrs.harvard.edu/urn-3:HUL.InstRepos:37171537

\section{Terms of Use}

This article was downloaded from Harvard University's DASH repository, and is made available under the terms and conditions applicable to Other Posted Material, as set forth at http:// nrs.harvard.edu/urn-3:HUL.InstRepos:dash.current.terms-of-use\#LAA

\section{Share Your Story}

The Harvard community has made this article openly available.

Please share how this access benefits you. Submit a story.

Accessibility 


\title{
Which findings should be published?*
}

\author{
Alexander Frankel ${ }^{\dagger} \quad$ Maximilian Kasy ${ }^{\ddagger}$
}

June 6, 2018

\begin{abstract}
Given a scarcity of journal space, what is the socially optimal rule for determining whether an empirical finding should be published? We show that if the goal of publication is to inform the public about a policy-relevant state of the world, then one should publish extreme results. For specific policy objectives, the optimal rule may take the form of a one- or two-sided test comparing the point estimate to the prior mean. Dynamic considerations may additionally justify the publication of precise null results. If one insists on a rule for which standard inference remains valid conditional on publication, however, the publication rule must not select on the study's findings (but may still select on the study's design).
\end{abstract}

KEYWORDS: Publication bias, mechanism design, value of information JEL CoDes: C44, D80, D83

\section{Introduction}

Not all empirical findings get published. Journals may be more likely to publish findings that are statistically significant, as documented in studies such as Franco et al. (2014), Brodeur et al. (2016), and Andrews and Kasy (2017). They may also be more likely to publish findings that are surprising, or conversely ones that confirm some prior belief. Whatever its form, selective publication distorts statistical inference. If only estimates with large effect sizes were to be written up and published, say, then published studies would systematically overstate true effects. Publication bias has been offered as one explanation for the perceived replication crisis in the social and life sciences 1

In response to these concerns, there have been calls for reforms in the direction of non-selective publication. One proposal is to promote statistical practices that de-emphasize statistical significance, for instance by banning "stars" in regression tables. Another proposal is for journals adopt Registered Reports, in which pre-registered analysis plans are reviewed and accepted prior to data

\footnotetext{
*We thank Isaiah Andrews, Victoria Baranov, Gary Chamberlain, Emir Kamenica, Matthew Notowidigdo, Jesse Shapiro, and Glen Weyl for helpful discussions and comments.

†University of Chicago Booth School of Business. Address: 5807 South Woodlawn Avenue Chicago, IL 60637. E-Mail: afrankel@chicagobooth.edu.

${ }^{\ddagger}$ Department of Economics, Harvard University. Address: Littauer Center 200, 1805 Cambridge Street, Cambridge, MA 02138. E-Mail: maximiliankasy@fas.harvard.edu.

${ }^{1}$ Worries about selective publication go back at least to Sterling (1959). Discussions of publication bias and other threats to the credibility and reproducibility of scientific output can be found in Ioannidis (2005), Ioannidis (2008), and in reviews including Simmons et al. (2011), Gelman and Loken (2014), and Christensen and Miguel (2016). Open Science Collaboration (2015) and Camerer et al. (2016) conduct large-scale replications of experimental studies in psychology and economics, giving insight into the extent to which published results are in fact reproducible.
} 
collection (see Nosek and Lakens (2014) or Chambers et al. (2014)). Registered Reports guarantee that publication will not select at all on findings, since after a plan is accepted, the journal is committed to publishing the study and the researcher has no flexibility over which results to write up.

In this paper we ask what the optimal rule is for determining whether a study should be published, given both its design and its findings. Our analysis is from an instrumental perspective: the value of a study is that it informs the public about some policy-relevant state of the world before the public chooses a policy action. In this framework, we will show that non-selective publication is not in fact optimal. Some findings are more valuable to publish than others. Hence, we will find a trade-off between policy relevance and statistical credibility.

In a world without constraints, it is immediate that the first-best policy would be for all results - or even better, all raw data - to be published. This paper solves for a second-best publication rule. We take as given that there is some constraint on the share of studies that will be published, or equivalently that there is some opportunity cost of publication. (After formally presenting the model, we discuss further interpretations of this cost, e.g., as arising from a public with limited attention.) If a submitted study is published, the public observes its findings and takes the optimal policy action given its updated belief. If not, the public never observes the study's results, and does not necessarily even know that a study was conducted; the public then takes a default action. This default action in the absence of publication is based on a default belief, where we allow for either a Bayesian public whose default belief correctly accounts for publication bias or for a naive public whose default belief always remains at its prior. The optimal publication rule is the one that maximizes the public's expected payoff from the eventual policy choice, minus the publication cost.

The optimal publication rule defined in this manner should select on a study's findings. To understand why, note that there is no instrumental value from publishing a study with a "null result" that doesn't move the policy away from the default action. The same policy would have been taken even if the study weren't published, so publishing would incur a cost without a benefit. The studies that are worth publishing are the ones that show that there is some payoff gain from taking an action other than the default.

For two canonical policy environments, we give precise characterizations of the optimal publication rules. In the first environment, the public makes a continuous policy decision, such as the choice of a tax rate, and has quadratic losses in matching the policy to its ideal point. The optimal publication rule then takes the form of a "two-sided test:" the journal publishes estimates that are sufficiently far above or below the prior mean. In the second environment, the public makes a binary policy choice, such as whether to implement a job training program. Here the journal uses a "one-sided test." For instance, in the absence of publication, the default action might be to not implement the program. The journal will then only publish studies with high estimates of the program's benefit, which convince the public to implement the program. Note that the critical values of these one- and two-sided tests come from a cost-benefit calculation, rather than being set to a conventional level such as $\mathrm{p}=.05$ against a null hypothesis of zero.

Moving beyond these two special cases, suppose that the public faces any "supermodular" policy decision in which it prefers to take higher policy actions in higher states of the world. In that setting 
we show that it is generally more socially valuable to publish studies with more extreme results that is, studies which lead to more extreme beliefs.

We wish to stress that the nature of our exercise is to solve for the socially optimal rule regarding whether to publish a study that has some given set of results. That is, we do not model the incentives of researchers or journals, and we are not attempting to characterize the equilibrium publication rule arising from a strategic interaction of these agents. As discussed in Glaeser (2006), researcher incentives play an important role in the publication process. Researchers make choices over the topics they study and their study designs, and then may selectively submit or possibly even manipulate their findings ${ }^{2}$ We do explore one way that researcher study design choices may respond to journal publication rules in Section 6 .

After characterizing optimal publication rules, we return to the distortions caused by selective publication. Consider a study that consists of a normally distributed point estimate paired with a standard error. It is immediate that common forms of inference are valid if the publication probability does not depend on the point estimate given the standard error. We show that the inverse is also true: Under any policy in which the publication probability depends on the point estimate, conditional on publication, common forms of frequentist inference will be invalid. Point estimates are no longer unbiased, for instance, and uncorrected likelihood-based estimation will not be accurate. Moreover, when a study is not published, a naive public that maintains its prior will have distorted beliefs relative to a Bayesian public that accounts for publication bias. If we desire that standard inference or naive updating be valid, we must impose a non-selective publication rule that does not depend at all on the point estimate (although we may still publish studies with small standard errors over studies with large standard errors).

Putting these results together, we see that selectively publishing extreme results is better for policy-relevance but leads to distorted inference. To the extent that the current (selective) publication regime qualitatively resembles the optimal rules we derive, then, a move towards non-selective publication in order to improve statistical credibility might have costs as well as benefits.

One abstraction in the model described above is that it considers a "static" environment with a single paper to be published and a single action to be taken. One may also be interested in the longer-term implications of publication rules, as in McElreath and Smaldino (2015) and Nissen et al. (2016) 3 To get some insight into these issues, we consider a dynamic extension to our model that appends a second period in which exogenous information arrives before another action is taken. The publication decision in the first period now affects the action in both periods. Just as before, we find a benefit of publishing extreme results. But we also find a new benefit of publishing precise results, even precise null results that don't change the current action. To wit, publishing a precise result today helps avoid future mistakes arising from the noise in the information that has yet to arrive. We show that publishing null results is most valuable when this future information is neither too

\footnotetext{
2 Furukawa (2018) looks at a model (without journals) in which researcher decisions to publish papers interact with a public policy choice, where in equilibrium researchers choose to publish papers with extreme results. Müller-Itten (2017) looks at a competition between journals for prestige, in which journals choose whether to publish a submitted study based on a signal of its quality while researchers choose which journal to submit to.

3 McElreath and Smaldino (2015) and Nissen et al. (2016) provide dynamic models to study whether an academic publication process with publication bias will eventually converge to truthful estimates. Akerlof and Michaillat (2017) performs a similar exercise for a more evolutionary form of the accumulation of academic knowledge.
} 
precise nor too imprecise.

Another abstraction in the above model is that it took the arrival of studies submitted to journals to be exogenous. We consider another extension in which researchers may alter their study designs in order to increase their chances of publication. Here, the researcher pays a cost to control the precision of a study she conducts, e.g., a higher cost for an experiment with a larger sample size. In response to these researcher incentives, we find that the journal should adjust the publication rule in two ways: the journal rejects imprecise studies regardless of their findings, and it becomes more willing to publish studies that are sufficiently precise. This new publication rule induces the researcher to conduct studies at an increased precision.

Throughout the paper, our derivations of optimal publication rules rely on characterizing the value of information for specified decision problems. Most theoretical treatments of the value of information study the ex ante value of an experiment, i.e., the expected value prior to the realization; see classic treatments in Blackwell (1953), Lehmann (1988), or Persico (2000). These ex ante comparisons are relevant for a characterization of non-selective publication rules, as we explore in Section 4.2 However, we generally allow for publication to select on the findings. We are therefore predominantly concerned with the ex post value of information given an experiment's realization, as studied in Frankel and Kamenica (2018). The decision to reveal a signal, at a cost, based on its realization is also related to the analysis of the discretionary disclosure of positive and negative news in Verrecchia (1983) and followup work.

The rest of the paper is structured as follows. Section 2 introduces our basic model of publication. Section 3 shows how to solve for the optimal publication rules, and provides some characterizations of the solution. Section 4 addresses the distortions that arise from selective rather than non-selective publication. Section 5 presents a two-period version of the model. Section 6 presents a model with researcher incentives. Finally, Section 7 concludes with additional discussion and extensions, such as considering optimal publication rules under objective functions that maximize something other than policy-relevance. Proofs are in the Appendix.

\section{The model of publication}

In Sections 2.1 2.3 we present our benchmark model of publication and in Section 2.4 we discuss interpretations of the publication cost.

\subsection{Timing of the game}

There is an uncertain state of the world whose value is relevant to some public policy decision. A study that reveals information about this state may or may not be submitted to a journal. If a study is submitted, the journal will decide whether to publish it, and the results of the study are observed by the public if the study is published. Then the public chooses a policy.

Let $\theta \in \Theta \subseteq \mathbb{R}$ denote the state of the world, and suppose that there is a common prior $\pi_{0}$ on this state shared by the public and the journal. The probability that a study arrives is $q \in(0,1]$, independent of $\theta$. A study can be summarized by the pair of random variables $(X, S)$. 
The variable $S$, with generic realization $s \in \mathcal{S}$, represents the study design and the variable $X$, with generic realization $x \in \mathcal{X}$, represents the study finding. The design $S$ is drawn according to a distribution $F_{S}$, independently of the state $\theta$. The finding $X$ is drawn from the distribution $F_{X \mid \theta, S}$, with pdf $f_{X \mid \theta, S}$ relative to some dominating measure. That is, the study design determines how the distribution of the finding depends on the state. A leading example that we will consider is that of a normally distributed signal, with $X$ in $\mathbb{R}, S$ in $\mathbb{R}_{++}$, and $X \mid \theta, S \sim \mathcal{N}\left(\theta, S^{2}\right)$.

If a study arrives, it will be evaluated by a journal which observes the finding and design $(X, S)$ and then decides whether to publish the study. The journal uses a publication rule $p: \mathcal{X} \times \mathcal{S} \rightarrow[0,1]$ where $p(X, S)$ describes the probability that a study $(X, S)$ is published. As a matter of terminology, we say that the journal or publication rule publishes a study when $p(X, S)=1$ and does not publish a study when $p(X, S)=0$. Let $D$ be the random variable with generic realization $d \in\{0,1\}$ indicating whether a study is ultimately published: $D=0$ if no study is published (because no study arrived, or because one arrived but was not published) and $D=1$ if a study arrived and is published.

After a study is published or not, the public's belief on $\theta$ updates to a posterior $\pi_{1}$. When no study has been published $(D=0), \pi_{1}$ is equal to some default belief $\pi_{1}^{0}$. When a study has been published $(D=1)$, its design $S$ and finding $X$ are publicly observed, and $\pi_{1}$ is instead equal to the belief $\pi_{1}^{(X, S)}$. We describe the belief updating process in Section 2.2 .

Finally, given updated beliefs $\pi_{1}$, the public takes a policy action $a \in \mathcal{A} \subseteq \mathbb{R}$ to maximize the expectation of a utility function $U: \mathcal{A} \times \Theta \rightarrow \mathbb{R} 4^{4}$ Let

$$
a^{*}\left(\pi_{1}\right) \in \arg \max \mathbb{E}_{\theta \sim \pi_{1}}[U(a, \theta)]
$$

indicate the chosen action when the public holds beliefs $\pi_{1}$. We assume existence of this argmax for any relevant utility functions and posterior distributions, and we confirm existence for all of our examples. Let $a^{0}=a^{*}\left(\pi_{1}^{0}\right)$ be the default action, i.e., the action taken under the default belief, whereas $a^{*}\left(\pi_{1}^{(X, S)}\right)$ is the action taken if a study $(X, S)$ is published.

Social welfare, corresponding to the shared objective of both the journal and public, is based on the action payoff net of a publication cost. Let $c>0$ indicate the social cost of publication; we discuss interpretations of this cost in Section 2.4. The realized welfare $W(D, a, \theta)$ induced by publication $D$, chosen action $a$, and state of the world $\theta$, is

$$
W(D, a, \theta)=U(a, \theta)-D c
$$

We take the study arrival and study design $S$ to be exogenous. (Section 6 explores an extension in which study arrival and design are endogenous to the publication rule.) The policy action $a$ is mechanical given induced beliefs $\pi_{1}$. Thus, the key decision in the model is the choice of publication rule $p$. We will search for the publication rule that maximizes the ex ante expectation of welfare, which we call the optimal publication rule.

\footnotetext{
${ }^{4}$ While we maintain the assumption that states $\theta$ and actions $a$ are real numbers in order to facilitate economic interpretations of our results, our solution approach in Section 3.1 will apply to general spaces $\Theta$ and $\mathcal{A}$.
} 


\subsection{Beliefs and belief updating}

Belief updating from the prior $\pi_{0}$ to the posterior $\pi_{1}$ depends on whether a study is published or not.

\section{Beliefs conditional on publication}

If a study $(X, S)$ is published, the posterior belief becomes $\pi_{1}=\pi_{1}^{(X, S)}$. We assume that $\pi_{1}^{(X, S)}$ is derived according to Bayes' Rule given the signal $X \sim F_{X \mid \theta, S}$. By Bayes' Rule, and since $\theta$ is independent of $S$, the density of $\pi_{1}^{(X, S)}$ relative to the prior $\pi_{0}$ is given by

$$
\frac{d \pi_{1}^{(X, S)}}{d \pi_{0}}(\theta)=\frac{f_{X \mid \theta, S}(X \mid \theta, S)}{f_{X \mid S}(X \mid S)},
$$

with $f_{X \mid S}$ the unconditional density of $X$ under study design $S$ and prior $\theta \sim \pi_{0}$.

While a journal is evaluating a paper for publication, we can think of $\pi_{1}^{(X, S)}$ as the journal's interim belief; the public adopts this belief if the paper is published, and reverts to $\pi_{1}^{0}$ otherwise.

\section{Default beliefs in the absence of publication}

If a study is not published - meaning that either no study arrived, or that a study did arrive but was not published - then the public updates to a default belief $\pi_{1}^{0}$. We consider two distinct possibilities for updating in the absence of publication. Bayesian updating is the sophisticated rule that accounts for any selection induced by the publication process; naive updating is the unsophisticated rule which fails to account for selection. While Bayesian updating is "correct" in the fully specified model, we consider naive updating to be in many cases a realistic description of updating in practice 5 Note that the default belief $\pi_{1}^{0}$ will depend on the publication rule $p$ under Bayesian updating, but will not depend on the publication rule under naive updating.

Bayesian updating is the updating rule for a public that understands the fully specified model of the world and correctly accounts for unpublished studies. When no study is published, the public understands that this event could have occurred because no study arrived (probability $1-q$ ) or because a study arrived (probability $q$ ) and was unpublished (conditional probability $1-p(X, S)$ given $X$ and $S$, with $\theta \sim \pi_{0}, S \sim F_{S}$, and $\left.X \sim F_{X \mid \theta, S}\right)$. The public then updates beliefs on $\theta$ to $\pi_{1}^{0}$ according to Bayes rule ${ }^{6}$ Denote the Bayesian default belief under publication rule $p$ by $\pi_{1}^{0, p}$; its density relative to the prior is given by

$$
\frac{d \pi_{1}^{0, p}}{d \pi_{0}}(\theta)=\frac{1-q \mathbb{E}[p(X, S) \mid \theta]}{1-q \mathbb{E}[p(X, S)]}
$$

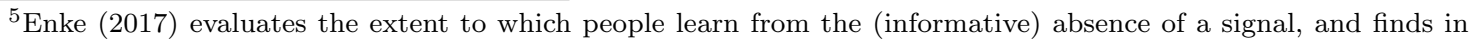
an experiment that subjects cluster around either fully accounting for selection or entirely neglecting it. More people can be prompted to account for selection by making the absence of a signal more salient, or by reducing cognitive distractions.

${ }^{6}$ If a publication rule publishes with probability one, and if the probability of study arrival $q$ is also one, then nonpublication is a zero probability event and beliefs are not pinned down by Bayes' rule. As a convention, in that case we let the Bayesian default belief be equal to the prior $\pi_{0}$.
} 
Naive updating is the updating rule for a public that ignores the possibility of unpublished studies. Under naive updating, the public's default belief when it does not see a publication, $\pi_{1}^{0}$, is equal to its prior: $\pi_{1}^{0}=\pi_{0}$. One can interpret a naive public as having an incorrect model of the world and, in the absence of seeing a publication, being "unaware" of the possibility that a study might have been submitted and rejected. Alternatively, this updating rule arises as the limiting Bayesian belief for the case of $q \rightarrow 0$, i.e., a fully rational public that did not expect a study to be submitted on this topic.

\section{Leading examples of priors and signals}

A typical state of the world $\theta$ estimated in an empirical economics study might be a demand or supply elasticity, the magnitude of a treatment effect, or the net benefit of implementing a program. Our leading example for a prior distribution will be the normal prior, in which case we take $\Theta=\mathbb{R}$ and $\pi_{0}$ given by $\mathcal{N}\left(\mu_{0}, \sigma_{0}^{2}\right)$, with $\mu_{0} \in \mathbb{R}$ and $\sigma_{0} \in \mathbb{R}_{++}$.

Our leading example for the signal distribution is that of normal signals. Under normal signals, we assume $\mathcal{X}=\mathbb{R}$ and $\mathcal{S} \subseteq \mathbb{R}_{++}$. The conditional distribution of findings, $F_{X \mid \theta, S}$, is given by $\mathcal{N}\left(\theta, S^{2}\right)$. The finding $X$ is interpreted as the point estimate for $\theta$, with standard error of $S$. $F_{S}$ then describes the ex ante distribution of standard errors.

When there is a normal prior and normal signals, the public's posterior belief on $\theta$ after publishing a study is given by

$$
\pi_{1}^{(X, S)}=\mathcal{N}\left(\frac{\sigma_{0}^{2}}{S^{2}+\sigma_{0}^{2}} X+\frac{S^{2}}{S^{2}+\sigma_{0}^{2}} \mu_{0}, \frac{S^{2} \sigma_{0}^{2}}{S^{2}+\sigma_{0}^{2}}\right) .
$$

Note that the study design $S$, as defined here, summarizes the informational content of the finding $X$. In the normal signals example, the variable $S$ in our model might be larger than a study's reported standard error, which only captures sampling variation. One additional source of error is from limited external validity, in which the parameter being estimated differs from and is only partially informative about the policy parameter of interest. Another source of error is a violation of the identifying assumptions required for the study's internal validity. We discuss some considerations that may arise when a journal is skeptical of reported standard errors in Section 7.1.

\subsection{Welfare maximization}

The value of publication in our model arises from a "policy relevance" objective: there is an instrumental value of publication in affecting policy decisions. The choice to publish rather than not publish a study $(X, S)$ has the direct effect, when that study is realized, of switching the policy

action from $a^{0}$ to $a^{*}\left(\pi_{1}^{(X, S)}\right)$ at a cost $c$. Under Bayesian updating, this choice may also have an indirect effect on the default action taken when no study is published.

The optimal publication rule $p(X, S)$ is the one that maximizes the ex ante expectation of welfare. To be precise, the ex ante expected welfare $E W\left(p, a^{0}\right)$ given a publication rule $p$ and a default action $a^{0}$ is

$$
E W\left(p, a^{0}\right)=\mathbb{E}\left[q p(X, S)\left(U\left(a^{*}\left(\pi_{1}^{(X, S)}, \theta\right)-c\right)+(1-q p(X, S)) U\left(a^{0}, \theta\right)\right],\right.
$$


where the expectation is taken with respect to $\theta \sim \pi_{0}, S \sim F_{S}$, and $\left.X \sim F_{X \mid \theta, S}\right]^{7}$ Under naive updating, the optimal $p$ maximizes expected welfare $E W\left(p, a^{*}\left(\pi_{0}\right)\right)$ over choice of $p$. Under Bayesian updating, the optimal $p$ maximizes $E W\left(p, a^{*}\left(\pi_{1}^{0, p}\right)\right)$ over choice of $p$.

\section{Leading examples of utility functions}

Two leading utility functions we consider are quadratic loss and binary action utility.

The quadratic loss utility function has $\mathcal{A}=\mathbb{R}$ and $U(a, \theta)=-(a-\theta)^{2}$. This is a canonical utility function for a public that makes a continuous policy decision $a$, with the state $\theta$ representing the public's uncertain ideal point. Under quadratic loss utility, the maximizing action choice given belief $\pi_{1}$ is $a^{*}\left(\pi_{1}\right)=\mathbb{E}_{\theta \sim \pi_{1}}[\theta]$. The subjective expectation of the action utility is $\mathbb{E}_{\theta \sim \pi_{1}}\left[U\left(a^{*}\left(\pi_{1}\right), \theta\right)\right]=$ $-\operatorname{Var}_{\theta \sim \pi_{1}}[\theta]$.

The binary action utility function has $\mathcal{A}=\{0,1\}$ and $U(a, \theta)=a \cdot \theta$. Here there is a binary decision, such as a choice to implement a program or not, where the state $\theta$ represents the net benefit of implementation. In that case $a^{*}\left(\pi_{1}\right)=\mathbf{1}\left(\mathbb{E}_{\theta \sim \pi_{1}}[\theta]>0\right)$ for $\mathbf{1}$ the indicator function (taking the action to be $a=0$ at indifference) and the subjective expectation of the action utility is $\mathbb{E}_{\theta \sim \pi_{1}}\left[U\left(a^{*}\left(\pi_{1}\right), \theta\right)\right]=\max \left\{0, \mathbb{E}_{\theta \sim \pi_{1}}[\theta]\right\}$.

We see that for both of these example utility functions, action choices depend only on mean beliefs. As a matter of notation, when working with either utility function, let $\mu_{0}, \mu_{1}^{0}$, and $\mu_{1}^{(X, S)}$ denote the means of the distributions $\pi_{0}, \pi_{1}^{0}$, and $\pi_{1}^{(X, S)}$.

\subsection{Interpretation of the publication cost}

As mentioned in the introduction, this paper begins with the observation that not all research findings get published. One can interpret our paper as solving for the optimal publication rule given any fixed share of studies to be published. Specifically, an optimization problem with a constraint on the share of studies that can be published would be equivalent to an unconstrained problem with an appropriate shadow cost of publication; we can take $c$ to be such a shadow cost.

Of course, there are real publication costs beyond any (presumably negligible) physical costs of printing and/or web hosting. On the journal side, the editor or referees may be responsible for verifying that the results are in fact as claimed. The editor can decide whether to desk reject the paper based on its claimed results. But if the editor wants to proceed, then before the paper can be published, peer reviewers have to put in work to confirm that the analysis behind these results is correct and should be trusted. On the researcher side, after one has determined the main results, there still is a cost of writing an article and preparing it for submission. These social costs would all be captured in the $c$ term.

In a more "reduced form" manner, the publication cost might also represent an opportunity cost of the public's attention. For a public with limited ability to process information, publishing one

\footnotetext{
${ }^{7}$ If the publication rule $p$ implies default belief $\pi_{1}^{0}$ under a specified updating rule, then the expected welfare it induces is $E W\left(p, a^{*}\left(\pi_{1}^{0}\right)\right)$. However, (4) also gives us the formula for calculating the counterfactual payoff from an arbitrary default action $a^{0}$ that may not be the one implied by $p$ and the updating rule. Note that we do not include anything related to $a^{*}\left(\pi_{1}^{(X, S)}\right)$ as an argument in $E W(\cdot)$ because $\pi_{1}^{(X, S)}$, and therefore $a^{*}\left(\pi_{1}^{(X, S)}\right)$, is given by a mechanical formula that does not depend on $p$ or the updating rule.
} 
study can pull attention from others. Relatedly, high-ranking journals do have a genuine scarcity of publication space and reject a large share of submissions (see Card and DellaVigna (2013)). So to the extent that publications in high-ranking journals receive disproportionate attention and influence, one can interpret our analysis as characterizing which papers should be published in these top journals 8

\section{Optimal publication rules}

This section begins with a general characterization of how to solve for optimal publication rules, covering both naive and Bayesian updating. We then apply this solution to derive optimal rules for our two leading example utility functions. Finally, we generalize the qualitative results to a broader class of utility functions.

\subsection{Solving the model}

Recall that after a paper has been submitted, the journal observes $(X, S)$ and has interim belief $\pi$ given by $\pi=\pi_{1}^{(X, S)}$. At this interim belief, the journal can evaluate the expected payoff from publication (leading to public belief $\pi_{1}=\pi$ and action $a^{*}(\pi)$ ) or from nonpublication (leading to public belief $\pi_{1}=\pi^{0}$ and action $\left.a^{0}\right)$. Denote by $\Delta\left(\pi, a^{0}\right)$ the gross interim benefit - that is, not including publication costs - of publishing a study that induces interim belief $\pi$ given default action $a^{0} 9$

$$
\Delta\left(\pi, a^{0}\right)=\mathbb{E}_{\theta \sim \pi}\left[U\left(a^{*}(\pi), \theta\right)-U\left(a^{0}, \theta\right)\right] .
$$

The objective is to maximize the ex ante welfare, not the interim welfare. But we can rewrite ex ante welfare $E W\left(p, a^{0}\right)$ of a publication rule $p$ and default action $a^{0}$ from (4) as the expected utility under the default action, plus the expected net interim benefit of publication:

$$
\begin{aligned}
E W\left(p, a^{0}\right) & =\mathbb{E}\left[q p(X, S)\left(U\left(a^{*}\left(\pi_{1}^{(X, S)}\right), \theta\right)-c\right)+(1-q p(X, S)) U\left(a^{0}, \theta\right)\right] \\
& =\mathbb{E}\left[U\left(a^{0}, \theta\right)\right]+q \mathbb{E}\left[p(X, S)\left(\Delta\left(\pi_{1}^{(X, S)} ; a^{0}\right)-c\right)\right]
\end{aligned}
$$

Say that publication rule $p$ is interim optimal given default action $a^{0}$ if it (almost surely) publishes a study when $\Delta\left(\pi_{1}^{(X, S)}, a^{0}\right)>c$ and does not publish when $\Delta\left(\pi_{1}^{(X, S)}, a^{0}\right)<c$. We see from 6 that welfare is separable across realizations of $X$ and $S$. Hence, fixing the default action $a^{0}$, a publication rule maximizes expected welfare $E W\left(p, a^{0}\right)$ over choice of $p$ if and only if it is interim optimal ${ }^{10}$

\footnotetext{
${ }^{8}$ One can make this "top journal" interpretation of our model formally precise up to a rescaling of costs. Assume that a study is first submitted to a top journal that has opportunity cost $c$ of publication, and if that journal does not publish the study then it ends up published in a low-ranked journal with publication cost 0 . The public will become aware of a study at a top journal with probability $\bar{r} \in(0,1]$, and a study at a low-ranked journal with probability $\underline{r}<\bar{r}$. The public's beliefs go to $\pi_{1}^{(X, S)}$ if they become aware of a study, and $\pi_{1}^{0}$ otherwise.

${ }^{9}$ Frankel and Kamenica 2018) provide general characterizations of these gross interim benefit functions - the value of information, in an ex post sense - across decision problems.

${ }^{10}$ In a game with different timing in which the journal could not commit to a publication rule, one might define a publication rule $p$ and default belief $\pi_{1}^{0}$ as constituting a "Bayes Nash equilibrium" if they jointly satisfy (i) $p$ is
} 
For concreteness, let $p^{I\left(a^{0}\right)}$ be the interim optimal publication rule given $a^{0}$ that deterministically publishes at indifference:

$$
p^{I\left(a^{0}\right)}= \begin{cases}1 & \text { if } \Delta\left(\pi_{1}^{(X, S)}, a^{0}\right) \geq c . \\ 0 & \text { otherwise }\end{cases}
$$

Under naive updating, the optimal publication rule solves $\max _{p} E W\left(p, a^{0}\right)$ subject to $a^{0}=$ $a^{*}\left(\pi_{0}\right)$. In particular, $a^{0}$ does not depend on $p$, so expected welfare is maximized by choosing an interim optimal publication rule. That is, a publication rule is optimal under naive updating if and only if it is interim optimal given default action $a^{0}=a^{*}\left(\pi_{0}\right)$.

Under Bayesian updating, the optimal publication rule solves $\max _{p} E W\left(p, a^{0}\right)$ subject to $a^{0}=$ $a^{*}\left(\pi_{1}^{0, p}\right)$. Solving this program requires taking into account the fact that $a^{0}$ changes with $p$. However, one can simplify the problem by observing that for any fixed $p$, the induced Bayesian default action $a^{0}=a^{*}\left(\pi_{1}^{0, p}\right)$ maximizes expected welfare $E W\left(p, a^{0}\right)$ over choice of $a^{0}$. This is because

$$
\begin{aligned}
\arg \max _{a^{0}} E W\left(p, a^{0}\right) & =\arg \max _{a^{0}} \mathbb{E}\left[(1-q p(X, S)) U\left(a^{0}, \theta\right)\right]=\arg \max _{a^{0}} \mathbb{E}\left[U\left(a^{0}, \theta\right) \mid D=0\right] \\
& =\arg \max _{a^{0}} \mathbb{E}_{\theta \sim \pi_{1}^{0, p}}\left[U\left(a^{0}, \theta\right)\right],
\end{aligned}
$$

where the last equality holds because the conditional distribution of $\theta$ given $D=0$ is exactly $\pi_{1}^{0, p}$, that from Bayesian updating. Therefore the Bayesian optimal publication rule $p$ equivalently solves $\max _{p} \max _{a^{0}} E W\left(p, a^{0}\right)$. This maximization program corresponds to a sequential game of common interest, with both players aiming to maximize the same objective. Moreover, $\max _{p} \max _{a^{0}} E W\left(p, a^{0}\right)=\max _{a^{0}} \max _{p} E W\left(p, a^{0}\right)=\max _{p, a^{0}} E W\left(p, a^{0}\right)$ : in a sequential game of common interest, the value is the same regardless of which player moves first, and is equal to the "planner's solution" maximizing the objective over the joint choice of $p$ and $a^{0}{ }^{11}$ Lemma 1 formally states this conclusion.

Lemma 1. Under Bayesian updating, let $p$ be an optimal publication rule and let $a^{0}=a^{*}\left(\pi_{1}^{0, p}\right)$ be the induced default action. Then for any publication rule $p^{\prime}$ and any action $a^{\prime}$, it holds that $E W\left(p, a^{0}\right) \geq E W\left(p^{\prime}, a^{\prime}\right)$.

One immediate implication from Lemma 1 and the above discussion is that even under Bayesian updating, $p$ is interim optimal given the induced default action. Hence, the optimal policy is interim optimal regardless of the updating rule:

Lemma 2. Under either naive or Bayesian updating, let $p$ be an optimal publication rule, $\pi_{1}^{0}$ be the induced default belief, and $a^{0}=a^{*}\left(\pi_{1}^{0}\right)$ be the default action. Then $p$ is interim optimal given $a^{0}$.

interim optimal given default action $a^{0}=a^{*}\left(\pi_{1}^{0}\right)$, and (ii) the default belief $\pi_{1}^{0}$ is equal to $\pi_{1}^{0, p}$, the one induced by Bayesian updating given the publication rule $p$. Our notion of optimality under Bayesian updating does not impose (i); nevertheless, Lemma 2 below clarifies that (i) will in fact be satisfied for an optimal publication rule. Hence, any optimal publication rule would induce a Bayes Nash equilibrium, but the converse need not hold.

${ }^{11}$ In a simultaneous game of common interest, the planner's solution is one equilibrium, but there may also be lower-payoff equilibria. 
Lemma 2 tells us that in searching for optimal publication rules, it is sufficient to consider only those rules that are interim optimal with respect to some default action. Indeed, at a default action $a^{0}$, it is without loss of generality to focus on the specific interim optimal rule $p^{I\left(a^{0}\right)}$ - all interim optimal publication rules give the same payoff $[12$ Going forward we adopt the convention of restricting attention to $p^{I\left(a^{0}\right)}$ in the class of interim optimal rules, and referring to this rule as "the" interim optimal publication rule given $a^{0}$.

The following result puts together how to solve for an optimal publication rule, summarizing the implications of Lemmas 1 and 2

\section{Proposition 1.}

1. Suppose that the updating rule is naive, in which case $\pi_{1}^{0}=\pi_{0}$ and $a^{0}=a^{*}\left(\pi_{0}\right)$. Then the interim optimal publication rule given this default action, $p^{I\left(a^{*}\left(\pi_{0}\right)\right)}$, is optimal.

2. Suppose that the updating rule is Bayesian, in which case $\pi_{1}^{0}=\pi_{1}^{0, p}$ and $a^{0}=a^{*}\left(\pi_{1}^{0, p}\right)$ under publication rule $p$.

(a) Let $\hat{a} \in \arg \max _{a \in \mathcal{A} \text { s.t. } a=a^{*}\left(\pi_{1}^{0, p^{I(a)}}\right)} E W\left(p^{I(a)}, a\right)$. Then the interim optimal publication rule given this default action, $p^{I(\hat{a})}$, is optimal.

(b) Let $\hat{a} \in \arg \max _{a \in \mathcal{A}} E W\left(p^{I(a)}, a\right)$. Then the interim optimal publication rule given this default action, $p^{I(\hat{a})}$, is optimal.

Proposition 1 part 1 formally restates the solution under naive updating. The optimal publication rule is the interim optimal rule given the naive default action.

For Bayesian updating, considered in Proposition 1 part 2 , we have a less direct characterization of the optimal publication rule. We provide two alternative maximization programs that can be solved to find the optimum. (Depending on the setting, one or the other may be more straightforward to apply.) Rather than maximizing over the original function space of publication rules, we are able to simplify the problem by maximizing over the action space, or a subset thereof. Specifically, each action induces an interim optimal publication rule, and the optimal publication rule is given by the induced interim optimal rule that yields the highest payoff.

To understand part 2a of Proposition 1, recall that just as each action induces an interim optimal publication rule, so too does each publication rule induce a Bayesian default action. Lemma 2 establishes that the optimal publication rule is interim optimal with respect to its induced default action. In other words, the default action is a "fixed point" of the mapping from actions to publication rules

\footnotetext{
${ }^{12}$ More precisely, $E W\left(p, a^{0}\right)$ is constant across all interim optimal $p$ for a given $a^{0}$. So for naive updating, where the default action is fixed in advance, the payoff of all interim optimal publication rules is the same. For Bayesian updating, the default action varies with the publication rule. However, for any optimal publication rule inducing some default action, it holds that all interim optimal publication rules given that same default action are also optimal - even if they induce different default actions. To see this, let $p$ be an optimal publication rule that induces default action $a^{0}$ (implying that $p$ is interim optimal given $a^{0}$ ). Let $p^{\prime}$ be any other interim optimal rule given $a^{0}-$ for instance, $p^{\prime}=p^{I\left(a^{0}\right)}-$ and let $a^{\prime}=a^{*}\left(\pi_{1}^{0, p^{\prime}}\right)$ be the default action induced by $p^{\prime}$. Then $E W\left(p^{\prime}, a^{0}\right) \geq E W\left(p, a^{0}\right)$ by interim optimality of $p^{\prime} ; E W\left(p^{\prime}, a^{\prime}\right) \geq E W\left(p^{\prime}, a^{0}\right)$ by the fact that $a^{\prime} \in \arg \max _{a} E W\left(p^{\prime}, a\right)$; and $E W\left(p, a^{0}\right) \geq E W\left(p^{\prime}, a^{\prime}\right)$ by Lemma 11 Hence, these inequalities are all equalities. The payoff from publication rule $p^{\prime}, E W\left(p^{\prime}, a^{\prime}\right)$, is equal to the payoff from the optimal publication rule $p, E W\left(p, a^{0}\right)$, and so $p^{\prime}$ is optimal as well.
} 
and back to actions. Therefore, when searching for an optimal publication rule, it is sufficient to maximize over interim optimal rules that are induced by some fixed point default action.

Unfortunately, solving for the set of fixed point default actions might not be straightforward. Part $2 \mathrm{~b}$ gives us a version of the result that does not require solving for fixed points. Instead, we can maximize over the full action space. Moreover, while the payoff of the publication rule $p^{I(a)}$ that is interim optimal with respect to action $a$ is generally given by $E W\left(p^{I(a)}, a^{*}\left(\pi_{1}^{0, p^{I(a)}}\right)\right)$ - requiring us to solve for the Bayesian default action induced by $p^{I(a)}$ - the proposition states that we need only evaluate the simpler expression $E W\left(p^{I(a)}, a\right)$. This is because, in the case that $a$ happens to be an action induced by some optimal policy, the payoff of the optimal policy $p^{I(a)}$ is in fact given by $E W\left(p^{I(a)}, a\right)$; see footnote 12 And in the case that $a$ is not an action induced by some optimal policy, the payoff $E W\left(p^{I(a)}, a\right)$ will be below that of the optimum; see Lemma 1. So this program recovers the correct maximum.

\subsection{Quadratic Loss Utility}

Under quadratic loss utility, welfare is $W(D, a, \theta)=-(a-\theta)^{2}-D c$ for $a \in \mathcal{A}=\mathbb{R}$. The public chooses an action equal to its posterior mean belief about the state. So when the default action is $a^{0}$, the gross interim benefit of publishing a study $(X, S)$ inducing belief $\pi_{1}^{(X, S)}$ with mean $\mu_{1}^{(X, S)}$ evaluates to $\left(\mu_{1}^{(X, S)}-a^{0}\right)^{2}$. The interim optimal publication rule is therefore

$$
p^{I\left(a^{0}\right)}(X, S)=\left\{\begin{array}{ll}
1 & \text { if }\left|\mu_{1}^{(X, S)}-a^{0}\right| \geq \sqrt{c} \\
0 & \text { otherwise }
\end{array} .\right.
$$

Lemma 2 establishes that this is also the form of the optimal publication rule for $a^{0}$ equal to $\mu_{1}^{0}$, the mean of the appropriate default belief. A study is published if and only if its results move the posterior mean by a sufficient amount in either direction relative to the default mean.

With naive updating, the optimal publication rule is given by plugging $a^{0}=\mu_{0}$ into (8); see Proposition 1 part 1 For Bayesian updating, the default action $a^{0}$ is endogenous to the publication rule. The following proposition provides a condition for which we can explicitly solve for the optimal publication rule under Bayesian updating, and indeed for which this optimal rule is the same as under naive updating.

Proposition 2. Suppose that there is quadratic loss utility, and that conditional on a study arriving the distribution of the interim mean $\mu_{1}^{(X, S)}$ is single-peaked and symmetric about the prior mean $\mu_{0} 13$ Then the optimal publication rule under Bayesian updating is the same as under naive updating: publish if and only if $\left|\mu_{1}^{(X, S)}-\mu_{0}\right| \geq \sqrt{c}$.

To show this result, we establish that under the single-peakedness and symmetry conditions, the prior mean is the only fixed point default action under Bayesian updating. So by Proposition 1 part 2a it must be the default action for the optimal policy.

\footnotetext{
${ }^{13}$ To be precise, by single-peaked and symmetric, we mean that (i) the distribution of the random variable $\mu_{1}^{(X, S)}$ has a pdf that is symmetric about $\mu_{0}$; and (ii) for any $\mu^{\prime}<\mu^{\prime \prime} \leq \mu_{0}$ it holds that if the pdf evaluated at $\mu^{\prime}$ is strictly positive, then the pdf evaluated at $\mu^{\prime \prime}$ is strictly larger than at $\mu^{\prime}$. (Symmetry implies the same result for $\mu_{0} \leq \mu^{\prime \prime}<\mu^{\prime}$.)
} 
Under the publication rule $p$ of Proposition 2, the induced Bayesian default belief $\pi_{1}^{0, p}$ will not be equal to the naive default belief $\pi_{0}$. However, they will both have the same mean, and therefore they lead to the same default action.

\section{Normal prior and normal signals}

We now explicitly characterize optimal publication rules under a normal prior and normal signals. This prior and signal structure satisfies the hypotheses of Proposition 2 the distribution of posterior means is single-peaked and symmetric about $\mu_{0}$ for each realized design $S$, and therefore across realizations of $S$ as well. Hence, the journal optimally publishes under either updating rule if and only if $\left|\mu_{1}^{(X, S)}-\mu_{0}\right| \geq \sqrt{c}$. Plugging in the updating formula (3):

Corollary 1. Suppose that there is a normal prior, normal signals, and quadratic loss utility. Then under either Bayesian or naive updating, the optimal publication rule publishes if and only if

$$
\left|X-\mu_{0}\right| \geq\left(1+\frac{S^{2}}{\sigma_{0}^{2}}\right) \sqrt{c} .
$$

This publication rule corresponds to a "two-sided test" in which the journal publishes if the point estimate is sufficiently high or sufficiently low. Equivalently, we can restate the publication rule in terms of a two-sided test for the t-statistic $\left(X-\mu_{0}\right) / S$ : the journal publishes if and only if

$$
\frac{\left|X-\mu_{0}\right|}{S} \geq\left(\frac{1}{S}+\frac{S}{\sigma_{0}^{2}}\right) \sqrt{c}
$$

See Figure 1

The form of a two-sided test is of course familiar from the null-hypothesis significance testing paradigm. However, we wish to highlight two distinctions of our policy from the two-sided test as it is traditionally applied. First, we compare the point estimate $X$ to the prior mean, not to some other point, e.g., a null hypothesis of $\theta=0$. Second, the cutoff for publication is not given by a rule of thumb such as a p-value of .05 or a t-statistic of 1.96 . The cutoff is determined by a cost-benefit analysis. We can take comparative statics on the value of this cutoff:

Corollary 2. Under the publication rule from Corollary 1 .

1. The publication cutoff $\left(1+\frac{S^{2}}{\sigma_{0}^{2}}\right) \sqrt{c}$ in terms of the difference of the point estimate from the mean is independent of the study arrival probability $q$ and the mean $\mu_{0}$. It is larger when the standard error $S$ is larger, the prior variance $\sigma_{0}^{2}$ is smaller, or the cost of publication $c$ is larger.

2. The publication cutoff $\left(\frac{1}{S}+\frac{S}{\sigma_{0}^{2}}\right) \sqrt{c}$ in terms of the magnitude of the t-statistic is nonmonotonic and convex in the standard error $S$ : it has a minimum at $S=\sigma_{0}$ and goes to infinity as $S \rightarrow 0$ or $S \rightarrow \infty$.

A given point estimate of $X$ moves beliefs more, and thus is published more (in the sense of a smaller cutoff value for $\left.\left|X-\mu_{0}\right|\right)$, when the standard error $S$ is smaller or when the prior uncertainty $\sigma_{0}$ is larger. Likewise, the journal publishes more when the cost of publication $c$ is lower. 
Figure 1: Optimal publication region (shaded) for quadratic loss utility, normal prior, normal signals.

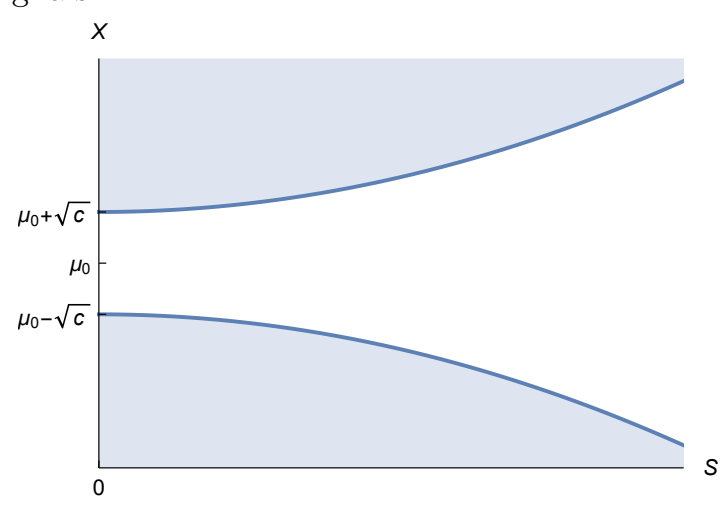

(a) In terms of the point estimate $X$, as a function of standard error $S$.

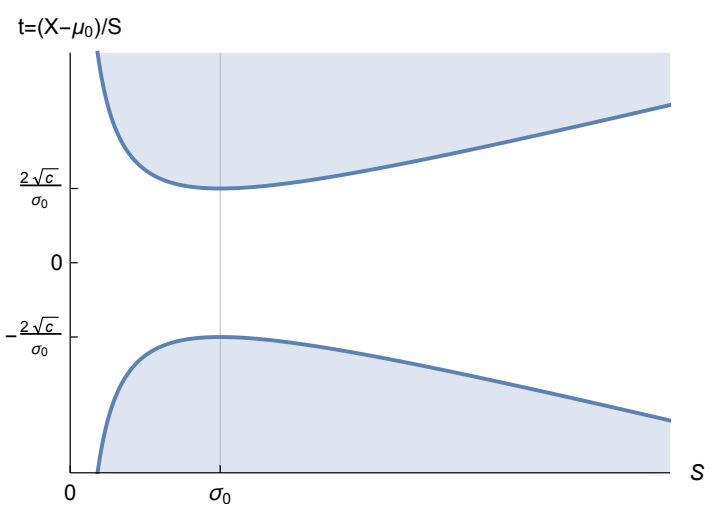

(b) In terms of the t-statistic $\left(X-\mu_{0}\right) / S$, as a function of standard error $S$.

We find something new for how the decision to publish at a given t-statistic depends on the standard error $S$. (For all other parameters, the comparative statics for publication in terms of the t-statistic would be identical to those on the point estimate.) For a precise study with a low standard error or an imprecise one with a high standard error, the journal requires a high t-statistic to be willing to publish; for a study of intermediate precision, the journal publishes at a lower t-statistic. To gain intuition, suppose the prior mean of $\theta$ is $\mu_{0}=0$ and the prior standard deviation is $\sigma_{0}=1$. The journal will publish a study if it moves the interim mean sufficiently far from 0 . Consider studies that might arrive with a given t-statistic $t=X / S$, say, $t=4$ (corresponding to a standard two-sided $\mathrm{p}$ value of $\lesssim .0001$ against a null of $\theta=\mu_{0}$ ). If a very precise study arrives with a t-statistic of 4 , then it must have had a small point estimate, and so it moves the mean very little: a study with a point estimate of $X=.04$ and a standard error of $S=.01$ moves the interim mean to $\simeq .039996$. As we begin to scale up the point estimate and standard error while keeping $t=4$, the mean moves higher: $X=4$ and $S=1$ leads to a mean of 2 . However, when we increase the point estimate and standard error further, the mean falls back towards 0 , because the result becomes too noisy to move beliefs much. With $X=400$ and $S=100$, the mean is back down to $\simeq .039996$. The journal would be most inclined to publish the middle result out of these three possibilities.

In other words, fixing the "statistical significance" as measured by the t-statistic, the change in mean first grows and then declines in the "practical significance" as measured by the magnitude of the point estimate. Generally, fixing a t-statistic $t=\left(X-\mu_{0}\right) / S$, the difference from the interim mean to the prior mean at standard error $S$ (with a corresponding point estimate of $X=\mu_{0}+\sigma_{0} t$ ) is given by $t \frac{\sigma_{0}^{2} S}{\sigma_{0}^{2}+S^{2}}$. The mean moves furthest at a standard error $S$ equal to $\sigma_{0}$, the standard deviation of the prior. The change in mean falls towards zero at high standard errors because the interim mean is a weighted average of the prior mean and the point estimate, with weights proportional to the inverse of the variances. While the point estimate increases linearly with the standard error at a given t-statistic, the weight on the point estimate decreases quadratically.

One could easily add in additional parameters to the model to get further comparative statics. 
For instance, consider the publication rule for research questions that are more or less "important." Modeling importance as a payoff coefficient $v$ in which $U(a, \theta)=-v \cdot(a-\theta)^{2}$, a more important policy with higher $v$ is mathematically equivalent to a lower $c$, i.e., a lower publication cost relative to the value of belief changes. So, the more important a state of the world is for policy, the more willing the journal should be to publish at a given point estimate or t-statistic. Similarly, if one decomposes the $S$ into a combination of sampling error and imperfect external validity, the journal is less willing to publish a point estimate when either the sampling error is higher or when the external validity is worse.

In Section 7.1 we note that the conclusion of Corollary 1, that it is optimal to publish extreme point estimates, could be reversed if the true standard error of a study were unobservable to the journal and the public. In that case extreme point estimates might be taken as a signal of large hidden noise. These "implausible" results would not move mean beliefs very much and would therefore not be worth publishing.

\subsection{Binary Action Utility}

Under binary action utility, welfare is given by $W(D, a, \theta)=a \theta-D c$ for $a \in \mathcal{A}=\{0,1\}$. The public chooses action $a=0$ if its posterior mean belief about the state is weakly less than 0 , and action $a=1$ if the posterior mean is positive. So when the default action is $a^{0}$, the gross interim benefit of publishing a study $(X, S)$ inducing belief $\pi_{1}^{(X, S)}$ with mean $\mu_{1}^{(X, S)}$ evaluates to 0 if either $a^{0}=0$ and $\mu_{1}^{(X, S)} \leq 0$, or $a^{0}=1$ and $\mu_{1}^{(X, S)} \geq 0$; and to $\left|\mu_{1}^{(X, S)}\right|$ otherwise. The interim optimal publication rule is therefore

$$
p^{I\left(a^{0}\right)}(X, S)=\left\{\begin{array}{cc}
1 \quad & \text { if } a^{0}=0 \text { and } \mu_{1}^{(X, S)} \geq c \\
& \text { or } a^{0}=1 \text { and } \mu_{1}^{(X, S)} \leq-c . \\
0 \quad & \text { otherwise }
\end{array}\right.
$$

Once again, Lemma 2 establishes that this is also the form of the optimal publication rule, for the appropriate default action.

Here, a study is published only if it moves the posterior mean belief sufficiently far in one direction. If the default action is low, then the journal only publishes studies that move beliefs up; if the default action is high, then the journal only publishes studies that move beliefs down. Contrasting to the quadratic loss utility function, the posterior mean must move sufficiently far not relative to the mean of the default belief, but relative to the belief - normalized to 0 - at which the public is indifferent between the two actions.

With naive updating, we get the optimal publication rule by plugging the naive default action into (9): $a^{0}=0$ if $\mu_{0} \leq 0$, and $a^{0}=1$ if $\mu_{0}>0$. See Proposition 1 part 1 . The following result gives a condition for the Bayesian optimal policy to be explicitly solvable and to be equal to the naive optimal policy. For the statement of the result, it is convenient to normalize the prior mean of $\theta$ to be less than zero, meaning that the naive default action will be $a^{0}=0$. The result then holds when the ex ante distribution of interim expectations on the state is sufficiently "left-leaning" relative to 
$\theta=0$. (An analogous result for $a^{0}=1$ and a sufficiently "right-leaning" distribution holds when the prior mean is above zero.)

Proposition 3. Let $\mu_{0} \leq 0$. Suppose that there is binary action utility, and that conditional on a study arriving the distribution of the interim mean satisfies $P\left(\mu_{1}^{(X, S)} \leq-k\right) \geq P\left(\mu_{1}^{(X, S)} \geq k\right)$ for all $k>0$. Then the optimal publication rule under Bayesian updating is the same as under naive updating: publish if and only if $\mu_{1}^{(X, S)} \geq c$.

The distributional assumption of Proposition 3 is strictly weaker than that of Proposition 2 . given a prior mean $\mu_{0} \leq 0$, any symmetric distribution of the interim mean $\mu_{1}^{(X, S)}$ is guaranteed to satisfy the condition of Proposition 3 even if it is not single-peaked. That said, we can not establish this result by applying Proposition 1 part 2a and showing that the naive default action is the unique Bayesian fixed point default action, as we did with Proposition 2 It can be the case - even under the stricter distributional conditions of Proposition 2 - that both actions are fixed points. It can be that with a low default action, the journal would only publish high signals, and after nonpublication the public would take a low action in response; while with a high default action, the journal would only publish low signals, and the public would take a high action in response. To show the result, we instead apply Proposition 1 part $2 \mathrm{~b}$. We directly confirm that the interim optimal publication rule with default action $a^{0}=0$ gives a higher payoff than with default action $a^{0}=1$.

Under this publication rule (and unlike the quadratic loss publication rule from Proposition 22, the mean of the induced Bayesian default belief $\pi_{1}^{0, p}$ will be lower than the mean of the naive default belief $\pi_{0}$. High results are published and low results are not, and so to a Bayesian the absence of publication is suggestive of a low state. Regardless, though, both default beliefs lead to the same default action of $a^{0}=0$ and thus the same (interim) optimal publication rule.

\section{Normal prior and normal signals}

We now explicitly characterize optimal publication rules under a normal prior and normal signals. Without loss of generality, assume that the prior mean is $\mu_{0} \leq 0$. This prior and signal structure satisfies the hypotheses of Proposition 3 meaning that the journal optimally publishes under either updating rule if and only if $\mu_{1}^{(X, S)} \geq c$. Plugging in the updating formula (3):

Corollary 3. Suppose that there is a normal prior with $\mu_{0} \leq 0$, normal signals, and binary action utility. Then under either Bayesian or naive updating, the optimal publication rule publishes a study if and only if

$$
X \geq\left(1+\frac{S^{2}}{\sigma_{0}^{2}}\right) c-\frac{S^{2}}{\sigma_{0}^{2}} \mu_{0} .
$$

This publication rule corresponds to a "one-sided test" in which a paper is published if the point estimate is sufficiently high. In terms of the t-statistic, the journal publishes if and only if

$$
\frac{X-\mu_{0}}{S} \geq\left(\frac{1}{S}+\frac{S}{\sigma_{0}^{2}}\right)\left(c-\mu_{0}\right)
$$

See Figure 2 
Figure 2: Optimal publication region (shaded) for binary action utility, normal prior, normal signals.

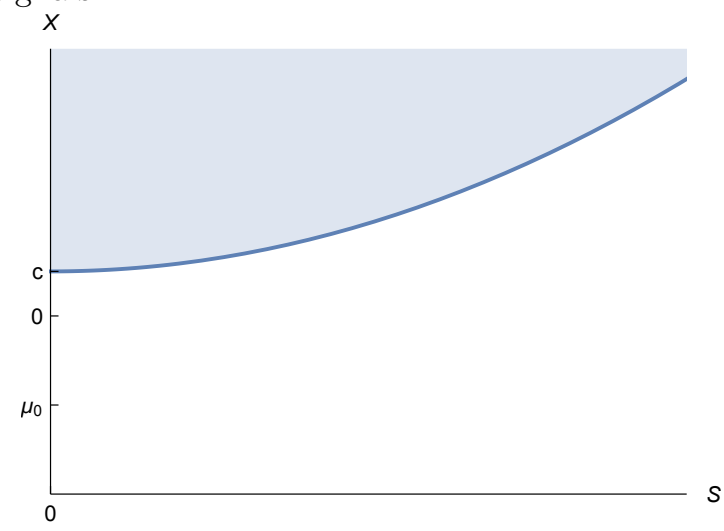

(a) In terms of the point estimate $X$, as a function of standard error $S$.

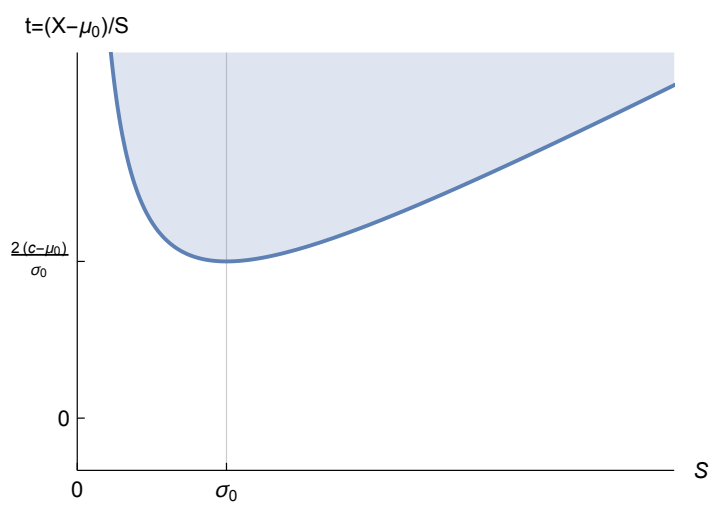

(b) In terms of the t-statistic $\left(X-\mu_{0}\right) / S$, as a function of standard error $S$.

Corollary 4. Under the publication rule from Corollary 3 ,

1. The publication cutoff $\left(1+\frac{S^{2}}{\sigma_{0}^{2}}\right) c-\frac{S^{2}}{\sigma_{0}^{2}} \mu_{0}$ in terms of the point estimate is independent of the study arrival probability $q$. It is decreasing in the mean $\mu_{0}$. It is larger when the standard error $S$ is larger, the prior variance $\sigma_{0}^{2}$ is smaller, or the cost of publication c is larger.

2. The publication cutoff $\left(\frac{1}{S}+\frac{S}{\sigma_{0}^{2}}\right)\left(c-\mu_{0}\right)$ in terms of the t-statistic is nonmonotonic and convex in the standard error $S$ : it has minimum at $S=\sigma_{0}$ and goes to infinity as $S \rightarrow 0$ or $S \rightarrow \infty$.

The comparative statics of publication with respect to the standard error $S$, the prior variance $\sigma_{0}^{2}$, and the cost of publication $c$ are essentially the same as those from the quadratic loss publication rule (see Corollaries 1 and 2). However, the two policies depend differently on the prior mean. Suppose we fix a point estimate $X>0$ and we consider prior means $\mu_{0}<0$. With quadratic loss utility, increasing $\mu_{0}$ towards 0 would make the journal less willing to publish: there will be a smaller difference $X-\mu_{0}$, and therefore the posterior mean will be closer to the prior mean. With binary actions, increasing $\mu_{0}$ towards 0 makes the journal more willing to publish: the posterior mean will be higher in absolute terms, indicating that the benefit of switching from $a=0$ to $a=1$ is higher.

\subsection{General utility functions}

In the above two examples of utility functions, we found that it was optimal to publish results that were in some sense "extreme" or "surprising" - ones that moved beliefs a lot. In this section we show that this conclusion generalizes beyond the examples of quadratic loss and binary action utility.

We start with a simple observation: if a study is a "null result," meaning that it does not move the optimal action away from the default, then the gross interim benefit of publication is zero. It can not be interim optimal to publish such a study. Therefore, the optimal publication rule never publishes null results. 
Observation 1. Fix a default action $a^{0}$ and an interim belief $\pi^{I}$. If $a^{*}\left(\pi^{I}\right)=a^{0}$, then $\Delta\left(\pi^{I}, a^{0}\right)=$ $0<c$.

Next, say that a utility function $U: \mathcal{A} \times \Theta \rightarrow \mathbb{R}$ is supermodular if for all $\underline{a}<\bar{a}$ and $\underline{\theta}<\bar{\theta}$, it holds that $U(\bar{a}, \bar{\theta})+U(\underline{a}, \underline{\theta}) \geq U(\underline{a}, \bar{\theta})+U(\bar{a}, \underline{\theta})$. Let $\geq_{F O S D}$ denote the first order stochastic dominance partial ordering on distributions. Under supermodular utility functions, the public takes higher actions when it believes that the state is higher (in the sense of FOSD); quadratic loss and binary action utilities are both supermodular.

Proposition 4 formalizes a sense in which it is generally optimal to publish extreme results when utility is supermodular.

Proposition 4. Let $U$ be supermodular. Let beliefs $\pi^{\prime}, \pi^{\prime \prime}$, and $\pi^{\prime \prime \prime}$ satisfy $\pi^{\prime \prime \prime} \geq_{F O S D} \pi^{\prime \prime} \geq_{F O S D} \pi^{\prime}$. Then for any default action $a^{0}$, it holds that $\Delta\left(\cdot, a^{0}\right)$ is quasiconvex in the sense that $\Delta\left(\pi^{\prime \prime}, a^{0}\right) \leq$ $\max \left\{\Delta\left(\pi^{\prime}, a^{0}\right), \Delta\left(\pi^{\prime \prime \prime}, a^{0}\right)\right\}$.

Suppose that some subset of study realizations leads to FOSD ordered beliefs. The proposition states that it is better to publish either a study that leads to a high belief or to a low belief than one that leads to an intermediate belief. By Lemma 2, then, the journal optimally publishes studies that lead to sufficiently low or high beliefs, but not beliefs in the middle 14 (See Appendix A.1 for examples illustrating how the conclusion of Proposition 4 can change when either supermodularity or FOSD ordering of beliefs is relaxed.)

Combining Proposition 4 and Observation 1 we further see that the published results will be ones that lead to extreme beliefs relative to the default. More precisely, fix some updating rule and some optimal publication policy leading to default belief $\pi_{1}^{0}$. Plugging the unpublished low belief $\pi^{\prime}=\pi_{1}^{0}$ into Proposition 4, we see that if the journal publishes a study leading to the higher belief $\pi^{\prime \prime}$, it must also publish one leading to the even higher belief $\pi^{\prime \prime \prime}$. Likewise, plugging in the unpublished high belief $\pi^{\prime \prime \prime}=\pi_{1}^{0}$, if the journal publishes the lower belief $\pi^{\prime \prime}$ then it also publishes the even lower belief $\pi^{\prime}$.

One sufficient condition for the interim belief from study realization $\left(x^{\prime \prime}, s^{\prime \prime}\right)$ to be FOSD higher than that of $\left(x^{\prime}, s^{\prime}\right)$ is for the realizations to satisfy the monotone likelihood ratio property (MLRP) in $\theta$. We say that $\left(x^{\prime \prime}, s^{\prime \prime}\right)$ and $\left(x^{\prime}, s^{\prime}\right)$ satisfy MLRP if the ratio $\frac{f_{X \mid \theta, S}\left(x^{\prime \prime} \mid \theta, s^{\prime \prime}\right)}{f_{X \mid \theta, S}\left(x^{\prime} \mid \theta, s^{\prime}\right)}$ is increasing in $\theta$. When findings $X=x$ at a given study design $S=s$ satisfy MLRP, we can apply Proposition 4 to derive the following corollary, which characterizes the study findings $X$ that should optimally be published. This result holds independently of any assumptions on the prior belief $\pi_{0}$.

Corollary 5. Fix either updating rule. let the utility function $U$ be supermodular. Furthermore, suppose that $\mathcal{X} \subseteq \mathbb{R}$, and that at some given study design $S=s$ it holds that for any $x^{\prime \prime}>x^{\prime}$ in $\mathcal{X},\left(x^{\prime \prime}, s\right)$ and $\left(x^{\prime}, s\right)$ satisfy MLRP. Then under the optimal policy, at $S=s$ a study is published if and only if $X$ lies outside of an interval. That is, given $s$, there exists an interval $I \subseteq \mathbb{R}$ such that $(X, s)$ is published if and only if $X \in \mathcal{X} \backslash I{ }^{15}$

\footnotetext{
${ }^{14}$ To be precise, no published study leads to beliefs that are in between those of two unpublished studies. But on a given chain of ordered beliefs, the journal might publish only high results, only low results, all results, or no results.

${ }^{15}$ We do not rule out that the interval $I$ may be empty, in which case all studies are published; or that $I$ may contain the full set $\mathcal{X}$, in which no studies are published.
} 
Under normal signals, for instance, for any $x^{\prime \prime}>x^{\prime}$ and any $s$, the realizations $\left(x^{\prime \prime}, s\right)$ and $\left(x^{\prime}, s\right)$ satisfy MLRP. So regardless of the prior, if there are normal signals and a supermodular utility function, the optimal publication rule at any given standard error will publish point estimates outside of an interval.

\section{Selective and non-selective publication}

In the previous section we characterized optimal publication rules from the perspective of policyrelevance. A key conclusion was that these welfare-maximizing publication rules tend to publish extreme findings and do not publish moderate findings. This conclusion contrasts with calls for reform aimed at eliminating selection, motivated by the statistical distortions and the lack of replicability that selective publication can cause.

We begin section 4.1 by reviewing how selective publication distorts standard inference in the framework of our model. Selective publication leads to biased estimators, size distortions of confidence sets and tests, and the invalidity of naive updating. This analysis complements earlier work on how standard inference from published results will be inaccurate when publication is based on a statistical significance filter, for instance in Rosenthal (1979) and Ioannidis (2005). We then show the novel result that, if we desire that standard inference be valid, the publication rule is not allowed to select on findings at all.

The fact that selective publication distorts inference means that there will be a trade-off between policy-relevance and credibility. The policy-relevance criterion pushes towards selectively publishing extreme results, while a desire to maintain the credibility of standard inference pushes instead towards non-selective publication. We conclude the section by characterizing the publication rules that maximize (policy-based) welfare subject to a constraint that publication may not select on findings.

\subsection{Publication bias}

Recall that the signal $X$ is drawn from the distribution $F_{X \mid \theta, S}$ with density $f_{X \mid \theta, S}$; our leading example was $X \mid \theta, S \sim N\left(\theta, S^{2}\right)$. Conventional statistical inference on $\theta$ from the estimator $X$ would be based on this distribution. However, under publication rule $p(X, S)$, the distribution of $X$ conditional on publication $(D=1)$ is different. The corrected density is

$$
f_{X \mid \theta, S, D=1}(x \mid \theta, s)=\frac{p(x, s)}{\mathbb{E}[p(X, S) \mid \theta, S=s]} \cdot f_{X \mid \theta, S}(x \mid \theta, s) .
$$

Inference that ignores the selectivity of the publication rule will lead to distorted conclusions.

To illustrate this point, consider the following example. Let $X$ be a normal signal with standard error of $S=1$, so $X \mid \theta \sim \mathcal{N}(\theta, 1)$. For this signal, the conventional unbiased estimator of $\theta$ would be $X$, and the conventional confidence set with a nominal $95 \%$ coverage probability would be $[X-$ 1.96, $X+1.96]$. But suppose that results are only published when $|X|>1.96$. Then, conditional on publication, $X$ is a biased estimator of $\theta$, and the interval $[X-1.96, X+1.96]$ does not have 
$95 \%$ coverage probability at every $\theta$. Indeed, the probability of $\theta \in[X-1.96, X+1.96]$ is actually equal to zero at $\theta=0$. The top left panel of Figure 3 plots the bias of $X$ as a function of $\theta$, defined as $\mathbb{E}[X-\theta \mid \theta, D=1]$, and the top right panel of Figure 3 plots the coverage probability $P(\theta \in[X-1.96, X+1.96] \mid \theta, D=1){ }^{16}$

Figure 3: Distortions due to selective publication.
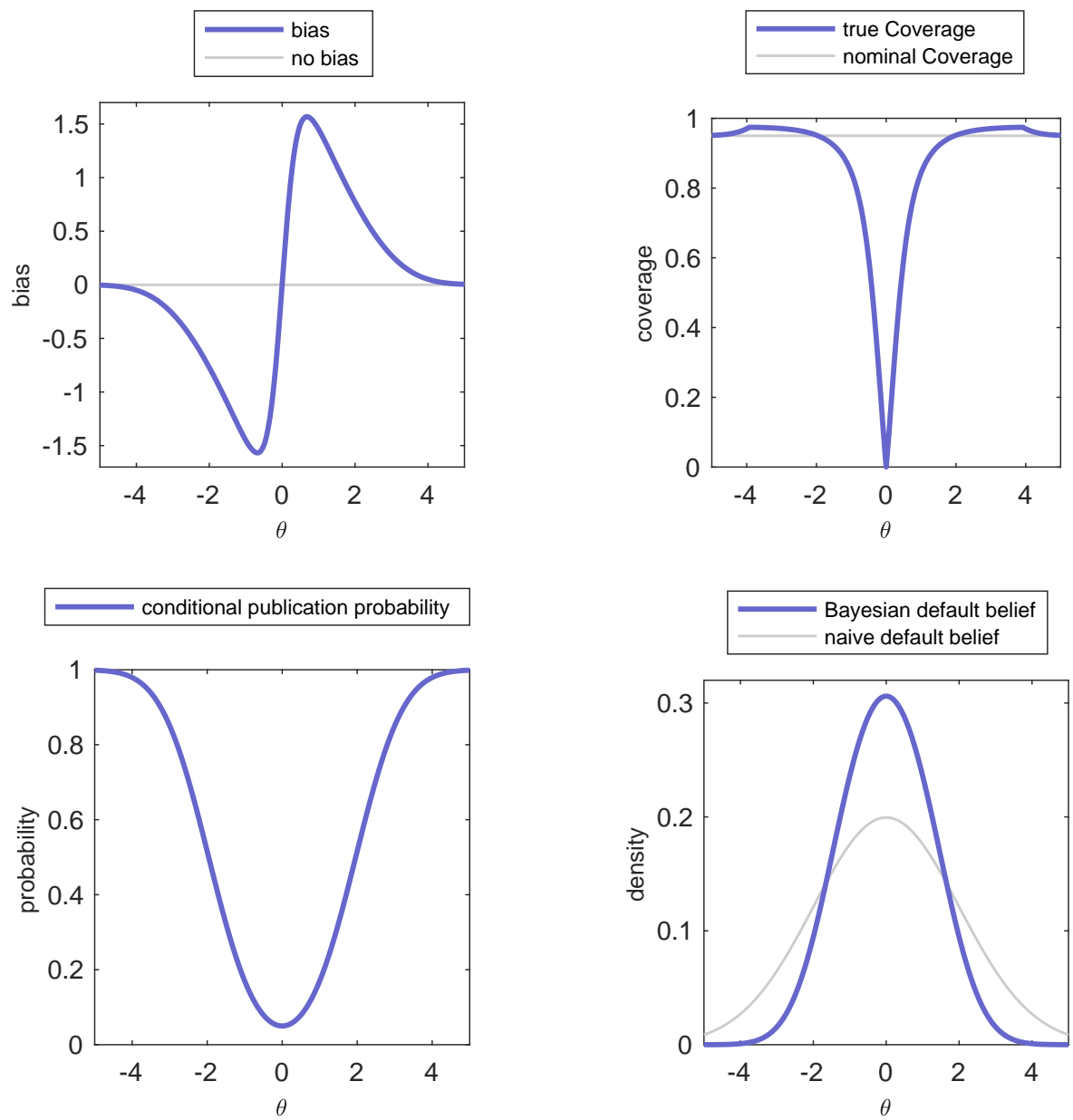

These plots are generated assuming $X \mid \theta \sim \mathcal{N}(\theta, 1)$ and that the conditional publication probability is given by $\mathbf{1}(X>1.96)$. The top left panel plots the bias of $X$ as an estimator of $\theta$, conditional on publication. The top right panel plots the coverage probability of $[X-1.96, X+1.96]$ as a confidence set for $\theta$, conditional on publication. The bottom left panel plots the probability of publication conditional on $\theta$ and on a study being submitted. The bottom right panel plots the Bayesian default belief relative to the naive default belief in the absence of publication, further assuming a prior of $\theta \sim \mathcal{N}(0,4)$ and a probability $q=1$ of study arrival.

Selective publication similarly impacts any form of likelihood-based inference. Maximum likelihood estimation conditional on $S$, likelihood ratio tests, and related methods must be adjusted for

\footnotetext{
${ }^{16}$ Similar figures for bias and coverage probability can be found in Andrews and Kasy (2017).
} 
selection if the ratio $f_{X \mid \theta, S, D=1}(x \mid \theta, s) / f_{X \mid \theta, S}(x \mid \theta, s)$ varies with $\theta$. As we see from 110, this ratio varies with $\theta$ whenever the publication probability $\mathbb{E}[p(X, S) \mid \theta, S=s]$ is not constant in $\theta$. For the example with $X \mid \theta \sim \mathcal{N}(\theta, 1)$ in which a study is only published if $|X|>1.96$, the publication probability falls as $\theta$ gets closer to 0 ; see the bottom left panel of Figure 3

Selective publication also implies that naive updating in the absence of publication yields distorted beliefs. In general, recall that the ex-ante probability of observing no publication conditional on $\theta$ is given by $1-q \cdot \mathbb{E}[p(X, S) \mid \theta]$. So the ratio of the Bayesian default belief to the prior is given by

$$
\frac{d \pi_{1}^{0, p}}{d \pi_{0}}(\theta)=\frac{1-q \cdot \mathbb{E}[p(X, S) \mid \theta]}{1-q \cdot \mathbb{E}[p(X, S)]} .
$$

The naive default belief (equal to the prior) differs from the Bayesian default belief whenever the publication probability $\mathbb{E}[p(X, S) \mid \theta]$ varies with $\theta$.

The bottom right panel of Figure 3 compares the density of the Bayesian default belief (the posterior absent publication) to that of the naive default belief for our running example, assuming a prior of $\theta \sim N(0,4)$ and a study arrival probability of $q=1$. When no publication is observed, a Bayesian who understands the data generating process knows that there may have been a study with $X \in[-1.96,1.96]$ that was submitted but went unpublished. Hence the Bayesian default belief places a higher probability on $\theta$ close to 0 , the center of the nonpublication interval, and a correspondingly lower probability on $\theta$ far from 0. Indeed, Abadie (2018) demonstrates how a failure to pass a standard statistical significance threshold can be extremely informative when studies are precise. Hence, if publication is based on a statistical significance test, the Bayesian default belief can greatly diverge from the naive one.

Our first result in this section formalizes the straightforward observation that, when publication does not select on findings, traditional inference goes through and naive updating is valid. Say that a publication rule $p$ is non-selective if $p(x, s)$ is constant in $x$ for each $s{ }^{17}$ Note that non-selective publication rules do not condition publication on the finding $X$, but they may condition publication on the design $S$, which is independent of the state.

Lemma 3. Suppose that the publication rule is non-selective and that $P(D=1)>0$. Then $f_{X \mid \theta, S, D=1}(x \mid \theta, s)=f_{X \mid \theta, S}(x \mid \theta, s)$, and thus the following properties hold.

1. Frequentist unbiasedness:

If functions $\widehat{g}: \mathcal{X} \times \mathcal{S} \rightarrow \mathbb{R}$ and $g: \Theta \times \mathcal{S} \rightarrow \mathbb{R}$ satisfy $\mathbb{E}[\widehat{g}(X, S) \mid \theta, S=s]=g(\theta, s)$ for all $\theta, s$, then $\mathbb{E}[\widehat{g}(X, S) \mid \theta, S=s, D=1]=g(\theta, s)$ for all $\theta, s$.

2. Frequentist size control:

Fix $\alpha \in(0,1)$ and fix a function $C$ from $\mathcal{X} \times \mathcal{S}$ to subsets of $\Theta$. If $P(\theta \in C(X, S) \mid \theta, S=s) \geq$ $1-\alpha$ for all $\theta, s$, then $P(\theta \in C(X, S) \mid \theta, S=s, D=1) \geq 1-\alpha$ for all $\theta, s$.

\footnotetext{
${ }^{17}$ Formally, we mean by this statement that $p(x, s)$ is constant in $x$ almost surely over realizations of $X$, i.e., that $P(p(X, s)=\mathbb{E}[p(X, s) \mid S=s] \mid \theta, S=s)=1$ for all $\theta$. Nothing changes if $p(x, s)$ may vary with $x$ on sets of $X$ that can only occur with zero probability given $\theta, S=s$.
} 
3. Publication probability constant in state:

The publication probability $\mathbb{E}[p(X, S) \mid \theta, S=s]$ is constant in $\theta$ for all $s$.

\section{Bayesian validity of naive updating:}

The Bayesian default belief $\pi_{1}^{0, p}$ is equal to the naive default belief, i.e., the prior $\pi_{0}$.

One interpretation of part 4 of the lemma, the Bayesian validity of naive updating, is as follows. Consider a "partially sophisticated" public which is aware that studies may sometimes be submitted and go unpublished, but which does not know the arrival rate of studies $q$ or the distribution of study designs $F_{S}$ (and may not even have a well-specified Bayesian prior over these objects). Such a public understands that naive updating can lead to distorted beliefs but it does not know how to correct this distortion. Under a non-selective publication rule, the public can in fact be confident in using naive updating: for any $q$ and any $F_{S}$, the Bayesian updating rule would be equal to the naive one.

Our next set of results establishes a sense in which, if one desires the above properties, then a publication rule cannot select on findings. For these results, we restrict to the class of normal signals in which $X \mid \theta, S \sim \mathcal{N}\left(\theta, S^{2}\right)$.

First, we show that if the point estimate is an unbiased estimator for $\theta$, or if the publication probability is constant in the state for every realization of $S$, then the publication rule must be non-selective. Likewise, if the public is not certain of the distribution of study designs $F_{S}$ and it seeks a publication rule for which naive updating is guaranteed not to yield distorted beliefs, then the publication rule must be non-selective ${ }^{18}$ Given that non-selective publication also implies these properties (Lemma 3), it follows that non-selective publication is equivalent to any of these three properties. We will return to the size control property below, in Proposition 6

Proposition 5. Suppose that there are normal signals and suppose that there is an open set $\Theta_{0} \subseteq \mathbb{R}$ contained in the support of the prior distribution of $\theta$. Then the following statements are equivalent:

1. Non-selective publication:

The publication decision $p(x, s)$ is constant in $x$ for each $s$.

2. Frequentist unbiasedness:

The expectation $\mathbb{E}[X \mid \theta, S=s, D=1]$ is equal to $\theta$ for all $\theta \in \Theta_{0}, s \in \mathcal{S}$.

3. Publication probability constant in state:

The publication probability $\mathbb{E}[p(X, S) \mid \theta, S=s]$ is constant over $\theta \in \Theta_{0}$ for all $s \in \mathcal{S}$.

4. Bayesian validity of naive updating:

For all distributions $F_{S}$ on $\mathcal{S}$, the Bayesian default belief $\pi_{1}^{0, p}$ is equal to the prior $\pi_{0}$.

Notice that, fixing a distribution $F_{S}$ of study designs, naive and Bayesian updating are equal as long as the publication probability is constant in the state unconditional on $S$. Part 3 of Proposition

\footnotetext{
${ }^{18}$ As discussed above, a partially sophisticated public might also be uncertain about $q$. However, the value of $q$ does not affect whether naive beliefs are distorted. The Bayesian default belief is equal to the prior for some given value of $q \in(0,1]$ if and only if it is equal to the prior for all values of $q \in(0,1]$.
} 
5 imposes a stronger condition, that the publication probability is constant conditional on any realization of $S{ }^{19}$ This stronger condition is equivalent to the requirement in part 4 that naive and Bayesian updating are equal not just for a given distribution $F_{S}$, but for all possible distributions $F_{S}$. That is the case in which a "partially sophisticated" public could confirm the validity of naive updating without knowing the distribution of study designs.

To prove that the latter three properties of Proposition 5 imply non-selective publication, we first establish that non-selective publication is implied by the publication probability being constant in the state (part 3). This result follows from the completeness of distributions in the normal location family; see, for instance, Theorem 6.22 in Lehmann and Casella (1998). One formulation of completeness for our setting is that for any fixed standard error $S=s$ and any function $g(x)$ for which $\mathbb{E}[g(X) \mid \theta, S=s]$ is constant in $\theta$ (over an open set), it holds that $g(x)$ is almost everywhere constant. Applying this result to the function $g(x)=p(x, s)$, we see that if the conditional publication probability at state $\theta$ is constant in $\theta$, then the publication probability $p(x, s)$ cannot vary with $x$, establishing non-selectivity. We then show that the Bayesian validity of naive updating implies a constant publication probability in the state (which holds for general signal structures), and that unbiasedness of the point estimate $X$ also implies a constant publication probability (which is a result more specific to normal signals).

Considering generalizations of these results beyond normal signals, note that completeness holds for all exponential families of full rank (Lehmann and Casella, 1998, Theorem 6.22). So a constant publication probability in the state would imply (and be equivalent to) non-selective publication for signal distributions $F_{X \mid \theta, S}$ derived not just from the normal but also, for instance, the Binomial, Poisson, Beta, Dirichlet, Chi-squared, and Gamma distributions.

For signal distributions outside of the exponential families, the result need not hold. For instance, consider a uniform signal distribution in which $X \mid \theta, S \sim$ Uniform $[\theta-S, \theta+S]$. Then any publication rule that is periodic with period $2 S$, such as $p(X, S)=0.5+0.5 \cdot \cos (\pi X / S)$, will have publication probability $\mathbb{E}[p(X, S) \mid \theta, S=s]$ constant over $\theta$ even though $p(x, s)$ varies with $x$. Moreover, even with normal signals, we require an open set of states for the results to hold. If the set of states is $\Theta=\{0,1\}$, then any publication rule that is symmetric in $X$ about $1 / 2$ will lead to a constant publication probability across the two states.

For the size control property, we get a weaker result than the equivalences of Proposition 5 . We show that any publication rule that publishes point estimates outside of an interval - as do the publication rules of Corollary 1 , Corollary 3 , and (more generally) Corollary 5 - will necessarily fail to control the size of confidence sets. In particular, conditional on publication, there will be some state $\theta$ for which the coverage probability of the standard confidence interval $[X-z S, X+z S]$ is lower than the nominal level of $\Phi(z)-\Phi(-z)$.

Proposition 6. Suppose that $\Theta=\mathbb{R}$, there are normal signals, and that the publication rule is given by $p(x, s)=\mathbf{1}(x \notin I(s))$ for some nondegenerate interval $I(s) \subsetneq \mathbb{R}$. Fix $z>0$. Then for any $s \in \mathcal{S}$,

\footnotetext{
${ }^{19}$ Here is an example with normal signals $\left(X \mid \theta, S \sim N\left(\theta, S^{2}\right)\right)$ in which publication probability is constant unconditionally on $S$ but not conditionally at each $S$. Suppose that $F_{S}$ places a $50 \%$ probability on $S=1$ and a $50 \%$ probability on $S=\sqrt{2}$. If (i) $S=1$, then let $\epsilon$ be an independent draw from $N(0,1)$ and publish if $X+\epsilon>0$; or (ii) if $S=\sqrt{2}$ then publish if $X<0$. (In other words, $p(X, 1)=\Phi(-X)$ and $p(X, \sqrt{2})=\mathbf{1}(X<0)$.) Then the probability of publication equals .5 for all $\theta$, but $p(x, s)$ is not constant in $x$ given $s$.
} 
there exists $\theta^{\prime} \in \Theta$ such that

$$
P\left(\theta^{\prime} \in[X-z \cdot s, X+z \cdot s] \mid \theta=\theta^{\prime}, S=s, D=1\right)<\Phi(z)-\Phi(-z) .
$$

The restriction to rules that publish outside of an interval is necessary for this result. In Appendix A.2 we show that for any fixed $z>0$ there do in fact exist other forms of selective publication rules for which the coverage probability of $[X-z S, X+z S]$ is equal to the nominal level. Note that if one required that the coverage probability of $[X-z S, X+z S]$ be equal to $\Phi(z)-\Phi(-z)$ for all $z$, rather for a single level of $z>0$, that would straightforwardly imply that the publication rule must be non-selective.

\subsection{Optimal non-selective publication rules}

One interpretation of Proposition 7 is that, under our leading example of normal signals, if we wish standard inference to remain valid, then we must restrict ourselves to non-selective publication rules. What is the optimal non-selective publication rule - the rule that maximizes the policymaker's utility subject to the constraint of being non-selective?

When the journal is restricted from screening on the point estimate $X$, the only remaining option is to screen on the standard error $S$. In that case, regardless of the prior or the utility function, the journal should publish studies with smaller standard errors over those with larger standard errors. The result follows from the fact that, conditional on a standard error $S=s$, it holds that $X \sim \mathcal{N}\left(\theta, s^{2}\right)$ is a Blackwell more informative signal of the state $\theta$ when $s$ is smaller.

Proposition 7. Suppose that there are normal signals. Then there exists $\bar{s} \geq 0$ for which the optimal non-selective publication rule publishes a study if and only if $S \leq \bar{s}$. The rule is the same under naive and Bayesian updating.

For $\bar{s}=0$ in Proposition 7, no study would be published, not even an arbitrarily precise one. Extending beyond normal signals, any time study designs $S$ can be ordered by Blackwell informativeness, the optimal non-selective publication rule would be to publish all studies with a sufficiently informative design.

Under a normal prior and quadratic loss utility - and maintaining the assumption of normal signals - we can explicitly solve for the optimal non-selective publication rule. If $\sigma_{0}^{2} \geq c$ (high prior uncertainty, low costs), then a study is published if $S \leq \bar{s}$, with $\bar{s}=\sigma_{0} \sqrt{\frac{\sigma_{0}^{2}}{c}-1}$; and if $\sigma_{0}^{2}<c$ (low prior uncertainty, high costs) then no study is published ${ }^{20}$ See Figure 4

\footnotetext{
${ }^{20}$ To work out this formula, let there be a normal prior, normal signals, and quadratic loss utility. The expected welfare if a study $(X, S)$ is published is $\operatorname{Var}_{\theta \sim \pi_{1}^{(X, S)}}[\theta]-c=\sigma_{0}^{2} \cdot \frac{S^{2}}{S^{2}+\sigma_{0}^{2}}-c$. Since this expression is independent of $X$, the expected welfare of publishing a study conditional on $S$ but unconditional on $X$ is $\sigma_{0}^{2} \cdot \frac{S^{2}}{S^{2}+\sigma_{0}^{2}}-c$. On the other hand, if a non-selective publication rule is used and no publication is observed, the default belief will be the prior $\pi_{0}$ for either updating rule. So the expected welfare of nonpublication, conditional on $S$ (which is uninformative about $\theta$ ) but unconditional on $X$, will be $\operatorname{Var}_{\theta \sim \pi_{0}}[\theta]=-\sigma_{0}^{2}$. The optimal non-selective publication rule publishes a study if $\sigma_{0}^{2} \cdot \frac{S^{2}}{S^{2}+\sigma_{0}^{2}}-c \geq-\sigma_{0}^{2}$, i.e., if $S^{2} \geq \sigma_{0}^{2}\left(\sigma_{0}^{2} / c-1\right)$.
} 
Figure 4: Optimal non-selective publication region (shaded) for quadratic loss utility, normal prior, normal signals.

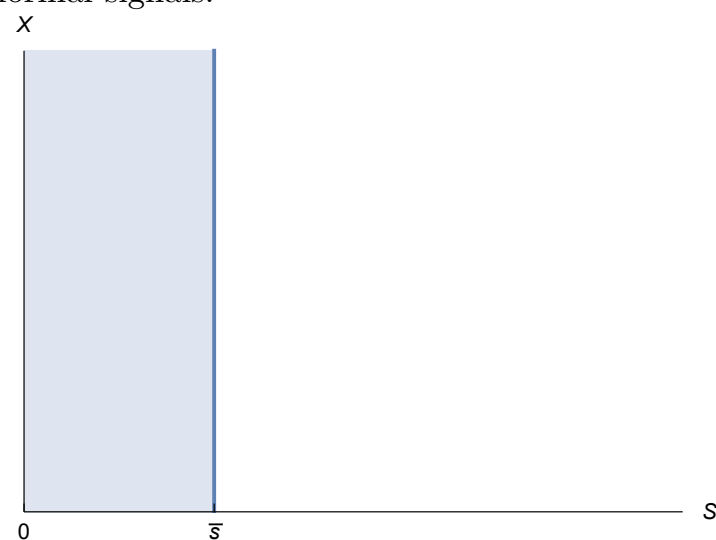

(a) In terms of the point estimate $X$, as a function of standard error $S$.

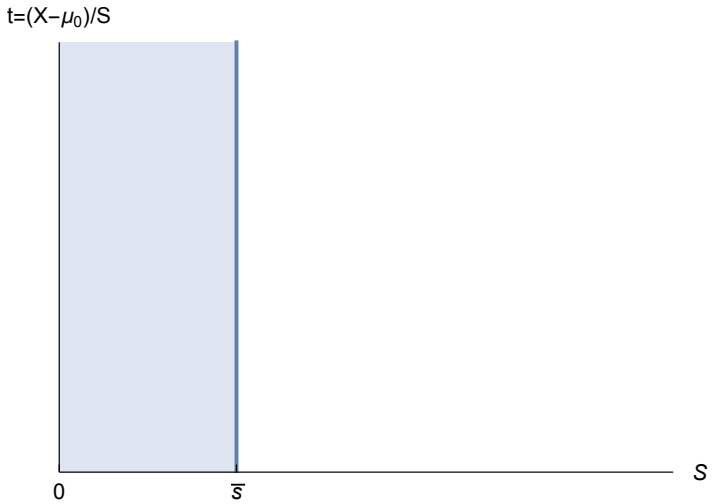

(b) In terms of the t-statistic $\left(X-\mu_{0}\right) / S$, as a function of standard error $S$.

If $c<\sigma_{0}^{2}$, as pictured, then for $\bar{s}=\sigma_{0} \sqrt{\sigma_{0}^{2} / c-1}$ a study is published if and only if $S \leq \bar{s}$. If instead $c \geq \sigma_{0}^{2}$, no studies are published.

\section{$5 \quad$ A dynamic model}

The model of Section 2 takes there to be a single source of information about the state of the world: a study that may be published or not. After the publication decision is made, a policy action is taken, and the game is over. If additional studies were to arrive in the future, though, the public might eventually receive information that convinced it to change its policy. The journal would have to make the decision of whether to publish a study today based on its expectations of what these future studies might reveal.

In order to explore some of these dynamic considerations, this section considers a two-period model. As before, there is an unknown policy-relevant state of the world $\theta$, which we take to be persistent over time. The original model of publication and policy choice is the first period of the game. The new second period captures, in reduced form, the impact of future studies: additional exogenous information arrives and the public takes another action ${ }^{21}$ That is, there is still just one publication decision to be made. But this decision must be made in the first period before the second period information is realized. If the study is published then it affects the belief, and therefore the actions, in both periods.

Set-up. At the start of the game, the common prior over $\theta$ is $\pi_{0}$. In the first period, a study is submitted to a journal with probability $q$. If the study arrives, it has finding and design $\left(X_{1}, S_{1}\right)$ with $S_{1} \sim F_{S_{1}}$ and $X_{1} \sim F_{X_{1} \mid \theta, S_{1}}$. The study is published with probability $p\left(X_{1}, S_{1}\right)$, and the public's induced belief is $\pi_{1}$. Belief updating to $\pi_{1}=\pi_{1}^{\left(X_{1}, S_{1}\right)}$ given publication outcome $D=1$ or to $\pi_{1}=\pi_{1}^{0}$ given publication outcome $D=0$ is as before, with the possibility of either naive or

\footnotetext{
${ }^{21}$ Solving a richer dynamic model with a sequence of publication decisions, in which the information revealed at later periods is endogenous to what has been previously published, is beyond the scope of this paper.
} 
Bayesian updating in the absence of a publication. Then the action $a_{1}$ is taken, with $a_{1}=a^{*}\left(\pi_{1}\right) \in$ $\arg \max \mathbb{E}_{\theta \sim \pi_{1}}[U(a, \theta)]$.

Next, in the second period, an exogenous signal $X_{2} \sim F_{X_{2} \mid \theta}$ is publicly observed. Beliefs update according to Bayes' Rule from prior $\pi_{1}$ to posterior $\pi_{2}$. (The information structure in period 2, summarized by $F_{X_{2} \mid \theta}$, is commonly known at the start of the game.) Finally, the action $a_{2}$ is taken, with $a_{2}=a^{*}\left(\pi_{2}\right) \in \arg \max \mathbb{E}_{\theta \sim \pi_{2}}[U(a, \theta)]$.

Social welfare is a weighted sum of action payoffs, minus a cost of publication $c>0$ if a study is published:

$$
W\left(D, a_{1}, a_{2}, \theta\right)=\alpha U\left(a_{1}, \theta\right)-D c+(1-\alpha) U\left(a_{2}, \theta\right) .
$$

The parameter $\alpha \in[0,1]$ describes the first-period payoff weight, relative to a $1-\alpha$ weight on the second period. The dynamically optimal publication rule $p$ maximizes the ex ante expectation of the above welfare.

In this section, we will restrict attention to quadratic loss utility. Moreover, we restrict attention to normal signals. As before, normal signals means that the first period signal takes the form $X_{1} \sim \mathcal{N}\left(\theta, S_{1}^{2}\right)$ for $X_{1} \in \mathbb{R}$, and $S_{1} \in \mathbb{R}_{++}$. It also now means that the second-period signal distribution is normal, with $F_{X_{2} \mid \theta}$ equal to $\mathcal{N}\left(\theta, s_{2}^{2}\right)$ for $s_{2} \in \mathbb{R}_{++}$.

Let us reiterate that, under this model, the standard error of the second-period signal $s_{2}$ is a parameter that is known at the start of the game. Our interpretation is that $s_{2}$ would be low (i.e., precise) when the journal expects that other high quality studies on the topic in question will soon be performed. The parameter $s_{2}$ would be high (i.e., imprecise) when the journal expects future studies on the topic to be performed infrequently, or to be of low quality.

Characterizing the optimal publication rule. We begin our analysis by deriving the dynamically optimal publication rule under normal priors and naive updating. For this special case we can get an explicit formula for the optimum; see an illustration of what this publication rule can look like in Figure 5

Proposition 8. In the two-period model with normal priors, normal signals, quadratic loss utility, and naive updating, the dynamically optimal publication rule is to publish a study $\left(X_{1}, S_{1}\right)$ if and only if the gross interim benefit is greater than or equal to $c$. The gross interim benefit is given by

$$
\frac{\sigma_{0}^{4}\left(s_{2}^{4}+2 \alpha \sigma_{0}^{2} s_{2}^{2}+\alpha \sigma_{0}^{4}\right)}{\left(\sigma_{0}^{2}+S_{1}^{2}\right)^{2}\left(\sigma_{0}^{2}+s_{2}^{2}\right)^{2}}\left(X_{1}-\mu_{0}\right)^{2}+(1-\alpha) \frac{\sigma_{0}^{8} s_{2}^{4}}{\left(\sigma_{0}^{2}+S_{1}^{2}\right)\left(\sigma_{0}^{2}+s_{2}^{2}\right)^{2}\left(\sigma_{0}^{2} S_{1}^{2}+\sigma_{0}^{2} s_{2}^{2}+S_{1}^{2} s_{2}^{2}\right)} .
$$

The dynamic benefit of publication expressed in 13 is a sum of two terms. The first term in (13) scales with $\left(X_{1}-\mu_{0}\right)^{2}$. This term gives a benefit of publishing extreme findings, similar to the interim benefit of publication from the single-period problem. These findings move the public's mean beliefs - and therefore its policy actions - further from the prior.

The second term in 13 expresses a benefit of publication that is novel to the two-period model. This term does not depend on $X_{1}$ and it is decreasing in $S_{1}$ : it gives a benefit of publishing precise results, independently of their point estimate. The value comes from the fact that publishing a precise result in period 1 can help reduce mistakes in period 2. (The benefit of publishing extreme 
Figure 5: Dynamically optimal publication region (shaded) for quadratic loss utility, normal prior, normal signals; naive updating.

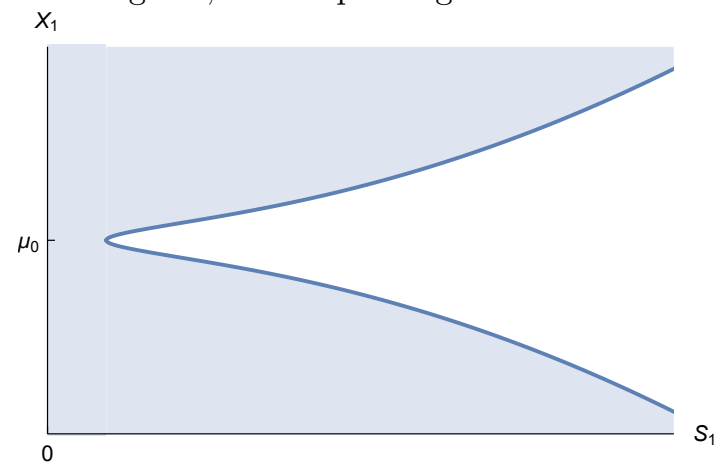

(a) In terms of the point estimate $X_{1}$, as a function of standard error $S_{1}$.

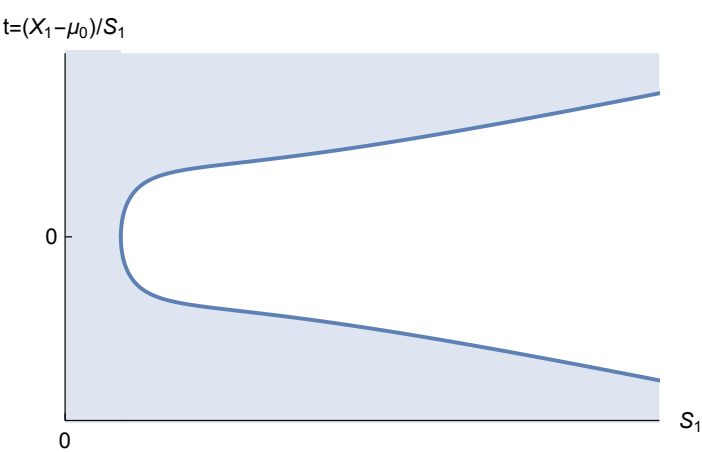

(b) In terms of the t-statistic $\left(X_{1}-\mu_{0}\right) / S_{1}$, as a function of standard error $S_{1}$.

As pictured, parameters are such that sufficiently precise results are published. Under different parameter values such as a higher cost $c$, a study with a null result of $X_{1}=\mu_{0}$ would not be published even with a perfectly informative design of $S_{1} \simeq 0$.

findings, given by the first term of (13), is also higher for more precise studies.)

To gain intuition on this benefit of publishing precise results, consider the benefit of publishing a "null result," i.e., a study with a point estimate exactly equal to the prior mean: $X_{1}=\mu_{0}$. Publishing such a study doesn't change the period 1 action payoff because the period 1 action will remain at $a_{1}=\mu_{0}$ either way. But there is a period 2 benefit. The period 2 posterior mean will be some convex combination of $\mu_{0}$ (the mean of $\pi_{1}$ ) and $X_{2}$. If the study at period 1 is not published then the belief $\pi_{1}$ will be less precise, leading to a lower weight on $\mu_{0}$ and a higher weight on $X_{2}$. And $X_{2} \sim \mathcal{N}\left(\theta, s_{2}^{2}\right)$ is an imperfect, noisy signal. Failing to publish a null result in period 1 therefore leads to excess variance of the second period action.

The following result gives comparative statics on the benefit of publishing null results.

Corollary 6. Under the hypotheses of Proposition 8, the gross interim benefit of publishing a result $\left(X_{1}, S_{1}\right)$ with $X_{1}=\mu_{0}$, given by

$$
(1-\alpha) \frac{\sigma_{0}^{8} s_{2}^{4}}{\left(\sigma_{0}^{2}+S_{1}^{2}\right)\left(\sigma_{0}^{2}+s_{2}^{2}\right)^{2}\left(\sigma_{0}^{2} S_{1}^{2}+\sigma_{0}^{2} s_{2}^{2}+S_{1}^{2} s_{2}^{2}\right)},
$$

is:

1. decreasing in $\alpha$, going to 0 as $\alpha \rightarrow 1$;

2. increasing in $\sigma_{0}$, going to 0 as $\sigma_{0} \rightarrow 0$;

3. decreasing in $S_{1}$, going to 0 as $S_{1} \rightarrow \infty$;

4. nonmonotonic and quasiconcave in $s_{2}$, approaching 0 as $s_{2} \rightarrow 0$ or $s_{2} \rightarrow \infty$.

The comparative static on $\alpha$ is straightforward. The benefit of publishing a null result - which increases payoffs only in the second period - is larger when the relative weight on the second period 
is larger. To understand the comparative statics on $\sigma_{0}$ and $S_{1}$, recall that the benefit arises from reducing second-period mistakes by making $\pi_{1}$ more precise relative to the prior $\pi_{0}$. There is a smaller benefit to increasing the precision of $\pi_{1}$ when the prior uncertainty (as measured by $\sigma_{0}$ ) is lower. And there is a larger increase in precision, and therefore a bigger benefit of publication, when the first period study is itself more precise (smaller $S_{1}$ ). Indeed, the journal might publish precise null results in the two-period model, but it will still not publish imprecise ones.

The more subtle part of Corollary 6 is the comparative static on $s_{2}$, the informativeness of the second-period signal, in part 4 . The benefit of publishing a null result is that it helps prevent the noisy signal $X_{2}$ from moving the public's mean belief to an incorrect value. But when the secondperiod signal is extremely precise $\left(s_{2} \simeq 0\right)$, there is no problem to be solved: the signal $X_{2}$ will reveal the state very precisely, and so to the extent that $X_{2}$ moves beliefs, it moves them to the truth. And when the second-period signal is extremely imprecise $\left(s_{2} \simeq \infty\right)$, there is also no problem: with high probability, observing $X_{2}$ will barely move beliefs. The period 2 studies that may cause mistakes by moving the public's belief to an incorrect value are those with an intermediate level of precision. In particular, the value of publishing a precise null is higher when $s_{2}$ is closer to the standard deviation of the prior uncertainty, $\sigma_{0}$.

Moving beyond naive updating, we do not have an explicit characterization of the dynamically optimal publication rule. But we can generalize the key implication of the two-period model in a way that does not depend on the updating rule: there is now a positive value of publishing any result that changes one's belief distribution from the default, even a null result that doesn't move the mean.

Proposition 9. Consider the two-period model with normal signals and quadratic loss utility. Fix a default belief $\pi_{1}^{0}$. Consider the gross interim benefit of publishing a study $\left(X_{1}, S_{1}\right)$ that induces period1 interim belief of $\pi_{1}^{\left(X_{1}, S_{1}\right)}$. For any fixed $s_{2}$, this benefit is strictly positive as long as $\pi_{1}^{\left(X_{1}, S_{1}\right)} \neq \pi_{1}^{0}$.

\section{$6 \quad$ A model with researcher incentives}

Thus far, we have taken submissions to the journal as exogenous. In reality submissions come about from a sequence of decisions by researchers: which topics to work on, what designs $S$ to choose, and which findings $X$ to actually write up and submit. In solving for an optimal journal publication rule, one ought to take into account the researchers' endogenous response to the incentives provided. To illustrate, this section presents a stylized model with incentives that explores a publication-motivated researcher's choices of whether to conduct a study and how to design that study.

Our analysis here complements some other recent theoretical investigations of how researcher or experimenter design choices may respond to incentives. In our example, the researcher's type will be commonly known and the design of a (submitted) study will be publicly observable, as in Henry and Ottaviani (2017) or the main analysis of McClellan (2017). Tetenov (2016) and Yoder (2018) study how a principal can screen across heterogeneous experimenters with privately known types. Libgober (2015) considers a setting in which study findings are observable, but the study design that led to a finding may be obscured. 
Set-up. There is a single researcher who takes a research topic - that is, a state of the world $\theta-$ as given. There is a common prior $\theta \sim \pi_{0}$ shared by all parties: the researcher, the journal, and the public.

The timing of the game is as follows. First, the journal publicly commits to a publication rule $p$ for studies on this topic. Then the researcher chooses whether to conduct a study and, if so, what study design $S$ to use; the researcher will submit the results of any study to a journal. Then the game proceeds as in Section 2 If a study $(X, S)$ is submitted it is published with probability $p(X, S)$, and finally the public updates its belief and takes a policy action. The key distinction from the original model is that the study submission probability $q$ and the distribution of study designs $F_{S}$ is now endogenous to the publication rule $p$.

To keep the analysis simple, we will restrict attention to naive updating. We will also focus on a normal signal structure, with $S \in \mathbb{R}_{++}$and $X \mid \theta, S \sim \mathcal{N}\left(\theta, S^{2}\right)$. Outside of the normal signal structure, our results would qualitatively hold for any class of signals in which study designs $S$ were ordered by Blackwell informativeness.

The researcher's problem. The researcher observes the publication rule $p$ and then decides whether to conduct a study. If she does conduct a study then she chooses its standard error $S \in$ $(0, \infty)$.

Normalize the researcher's outside option payoff from not conducting a study to 0 . If a study is conducted, the researcher values having it be published but pays a cost that depends on the precision of the study. Specifically, the researcher gets a benefit of 1 for getting a study published, independently of the study's results. The researcher pays a cost $\kappa(S)$ for conducting a study with standard error $S$, with $\kappa:(0, \infty) \rightarrow \mathbb{R}_{+}$. (Assumptions such as $\kappa^{\prime}(S)<0$ would be natural - the researcher pays more for an experiment with a larger sample size, say - but we do not actually need to impose any conditions on the cost function for the results that follow.) So the researcher's ultimate payoff if she conducts a study with standard error $S$ and publication outcome $D$ is

$$
D-\kappa(S)
$$

Denote the researcher's expected payoff from conducting a study with standard error $S=s$, given journal publication rule $p$, by $V(s, p)$ :

$$
V(s, p)=\mathbb{E}_{\theta \sim \pi_{0}, X \sim \mathcal{N}\left(\theta, s^{2}\right)}[p(X, s)]-\kappa(s) .
$$

The researcher's participation constraint for being willing to conduct a study is

$$
\max _{s \in(0, \infty)} V(s, p) \geq 0
$$

where we assume that the maximum is attained. Conditional on conducting a study, the researcher's 
choice of standard error $S$ is determined by the incentive compatibility condition

$$
S \in \arg \max _{s \in(0, \infty)} V(s, p)
$$

As before, we will assume that an argmax exists for any relevant $p$, without giving explicit conditions on primitives to guarantee that this will be the case.

The journal's problem. Let the journal maximize the expectation of welfare $W$ given by the policy payoff minus any cost of publication:

$$
W=U(a, \theta)-D c
$$

That is, we suppose that the journal does not place any weight on the researcher's utility. Furthermore, assume that the public updates naively, so that the public's default action is fixed at $a^{0}=a^{*}\left(\pi_{0}\right)$.

The journal's objective function takes the same form as in the original model, with the key distinction that the arrival of studies is no longer exogenous to the publication rule $p$. First, the study submission probability $q$ depends on $p: q=1$ if the participation constraint $(\mathrm{P})$ is satisfied, and $q=0$ otherwise. Second, conditional on participation, the standard error $S$ depends on $p$ through the incentive compatibility condition (IC). As is standard, assume that the researcher resolves indifferences in favor of the journal's preferences. The journal's problem is to choose an incentive-optimal publication rule $p$ that maximizes expected welfare subject to these endogenous responses.

Observe that, conditional on the arrival of a study, the journal's gross interim benefit of publication is unchanged from its earlier definition in (5). A study that induces a journal interim belief of $\pi_{1}^{(X, S)}$ when the public's default action is $a^{0}=a^{*}\left(\pi_{0}\right)$ yields gross interim benefit of $\Delta\left(\pi_{1}^{(X, S)}, a^{*}\left(\pi_{0}\right)\right)$.

In the original model with exogenous study submission, the journal's optimal policy was given by $p^{I\left(a^{*}\left(\pi_{0}\right)\right)}$, the interim-optimal publication rule in which a study $(X, S)$ is published if and only if $\Delta\left(\pi_{1}^{(X, S)}, a^{*}\left(\pi_{0}\right)\right) \geq c$. Let us impose the assumption that the researcher would in fact be willing to participate if the journal were to use this interim-optimal publication rule and would submit a study with $S=s^{\text {int }}$. This assumption will simplify both the solution and the exposition of our results.

Assumption 1. The participation constraint $(\mathrm{P})$ is satisfied under the interim-optimal publication rule $p=p^{I\left(a^{*}\left(\pi_{0}\right)\right)}$. Let $s^{\text {int }} \in \arg \max _{s} V\left(s ; p^{I\left(a^{*}\left(\pi_{0}\right)\right)}\right)$ be the researcher's choice of study design in response to the interim-optimal publication rule.

\section{Characterizing the optimal publication rule.}

Proposition 10. Consider the model with incentives under normal signals and naive updating, and suppose that Assumption 1 holds. Then there exist $\bar{s} \leq s^{\mathrm{int}}, \lambda \geq 0$, and $\rho \in[0,1]$ such that the 
following rule $p$ is incentive-optimal:

$$
p(X, S)=\left\{\begin{array}{c}
1 \quad \text { if } S=\bar{s} \text { and } \Delta\left(\pi_{1}^{(X, S)}, a^{*}\left(\pi_{0}\right)\right)>c-\lambda, \\
\quad \text { or if } S<\bar{s} \text { and } \Delta\left(\pi_{1}^{(X, S)}, a^{*}\left(\pi_{0}\right)\right) \geq c \\
\rho \quad \text { if } S=\bar{s} \text { and } \Delta\left(\pi_{1}^{(X, S)}, a^{*}\left(\pi_{0}\right)\right)=c-\lambda \\
0 \quad \text { otherwise }
\end{array} .\right.
$$

Given this rule, the researcher chooses to conduct a study with $S=\bar{s}$.

The form of the optimal rule - at least at the chosen study design $S=\bar{s}$ - is very similar to the interim-optimal rule that was used in the model without incentives. A study is published if the gross interim benefit is sufficiently high.

However, the journal distorts publication in two ways from the interim-optimal rule. First, the journal does not publish any studies with standard error $S>\bar{s}$. The researcher is therefore induced to invest additional resources into the precision of studies and to reduce $S$ from $s^{\text {int }}$ to $\bar{s}$. Second, at $S=\bar{s}$ the journal relaxes the interim benefit threshold for publication from $c$ to $c-\lambda$ in order to encourage researcher participation. Without that relaxation, a researcher might decide that a study at $S=\bar{s}$ would be too costly to conduct given its low likelihood of being published. (While in equilibrium the researcher never chooses $S<\bar{s}$, the journal has no reason to distort the publication rule at those more precise designs.)

In the original model without incentives, a journal which internalized all costs and benefits of publication would not need commitment power: ex ante payoffs were maximized by publishing according to what was interim-optimal after receiving a study. Having added researcher incentives, the two distortions now require two forms of journal commitment. The journal commits not to publish imprecise studies, even if such a study was conducted and turned out to have extremely striking results. This commitment is never actually tested on the equilibrium path, though - imprecise studies are not conducted. The journal also commits to publish studies with weak findings when they have the appropriate precision. This second form of commitment is tested, as these studies are submitted (and published) in equilibrium.

One key simplification of this model of incentives was the assumption that there was no heterogeneity across researchers. This fact was what guaranteed that researchers would always choose to conduct a study with a single standard error, known in advance. In a richer model, we would expect publication rules to reward more precise studies with higher publication probabilities in a more continuous manner than what we found here.

\section{$7 \quad$ Further Extensions}

In Sections 2, 6, we analyzed optimal publication rules supposing that the social value of publication was derived from a policy-relevance objective. Broadly speaking, we argued for the publication of extreme results. Extreme results are more valuable to publish than moderate results because they move public beliefs, and therefore public policies, further from the "default" that occurs in the 
absence of publication. To conclude the paper, we discuss how our analysis might change if we were to reconsider some of our main assumptions.

Section 7.1 considers the possibility that study designs may not be perfectly observed. Among other things, we argue that one may now want to qualify the earlier claim about publishing extreme results: it would still be optimal to publish results that moved beliefs further, but those results might not be the ones with the most extreme point estimates.

Next, Section 7.2 looks for publication rules that maximize social objectives other than policybased welfare. When the social objective is to learn the true state of the world, independently of any policy problem, we show that the form of publication rule may be essentially unchanged from our earlier analysis. However, when the social objective is to publish accurate results that are as close as possible to the truth, the publication rule can flip; the journal now wants to publish moderate rather than extreme results.

\subsection{Imperfectly observed study designs}

In determining whether to publish a study, a journal cares about the study's true information content. It may not be enough just to look at the reported standard error and treat that as the variable $S$ in our model of normal signals. As previously discussed, one concern is external validity: the parameter being estimated in the study may only be a proxy for the policy parameter of interest. Another concern is that the study may be internally flawed: a study with a misspecified model or an unconvincing identification strategy may report a very small standard error without actually being close to the truth.

When the study design is imperfectly observed, the point estimate can itself be informative as to the study's precision. To be concrete, assume that there are normal priors with mean normalized to 0 and there are normal signals, so that $\theta \sim \mathcal{N}\left(0, \sigma_{0}^{2}\right)$ and $X \sim N\left(\theta, S^{2}\right)$. But now assume that the realization of $S \sim F_{S}$ is unobserved by the journal and the public. As noted in Subramanyam (1996), observing a point estimate with a larger magnitude $|X|$ leads to higher beliefs on the unobserved noise $S$. In our application, a small point estimate would suggest that the study design was precise, while a large point estimate would be suggestive of some hidden noise. The extreme realization might be attributed to a violation of the identifying assumptions, to a coding error, or to some other unseen flaw.

Continuing the example with $X$ but not $S$ observed by the journal and public, and with $\theta \sim$ $\mathcal{N}\left(0, \sigma_{0}^{2}\right)$ and $X \sim N\left(\theta, S^{2}\right)$, further suppose that there is quadratic loss utility. The journal makes a publication decision based on the posterior mean of $\theta$, now conditional on $X$ but not $S$ :

$$
\mathbb{E}[\theta \mid X]=\mathbb{E}[\mathbb{E}[\theta \mid X, S] \mid X]=\mathbb{E}\left[\frac{\sigma_{0}^{2}}{S^{2}+\sigma_{0}^{2}} \mid X\right] \cdot X
$$

A higher belief on $S$ due to a larger point estimate $|X|$ translates into a lower weight $\mathbb{E}\left[\frac{\sigma_{0}^{2}}{S^{2}+\sigma_{0}^{2}} \mid X\right]$ on

the point estimate. Indeed, when the prior on $S$ is sufficiently dispersed, $\mathbb{E}\left[\frac{\sigma_{0}^{2}}{S^{2}+\sigma_{0}^{2}} \mid X\right]$ can decrease fast enough that $\mathbb{E}[\theta \mid X]$ is nonmonotonic and falls to 0 as $X$ goes to infinity. (In addition to Subramanyam (1996), see discussion of this issue in Dawid (1973), O'Hagan (1979), and Harbaugh 
et al. (2016).) An intermediate point estimate would therefore move an observer's mean belief more than a very large, "implausible," point estimate would. Let us restate that our results in Section 3 support publishing "extreme results" in the sense of results that lead to extreme beliefs. If extreme signal realizations are written off as implausible, then they would not lead to extreme beliefs and thus should not be published.

A related possibility is that the study design $S$, capturing the true informational content of the study's findings, is better observed by the journal than by the public. After all, the journal editor and referees are experts who are charged with carefully evaluating the quality of a paper; a policymaker reading the study might not have this expertise. Consider a model where the journal observes $(X, S)$ when making a publication decision, while if a paper is published the public sees only $X$. In such a model, the public can make on inference on the quality of the study design from the fact that the study was published. Publication implies that the journal had chosen to certify the study as clearing the bar of peer review. Suppose additionally that even unpublished studies are publicly available as working papers or preprints. In this case the only role of "publication" by a journal is certification or signaling value. A formal analysis of optimal publication rules in such an environment to be an interesting topic for future research.

\subsection{Alternative objectives}

\subsubsection{A Learning Objective}

Separate from any decision problem, the public might value more precise knowledge of the state of the world out of purely academic interest. One natural way of measuring the precision of beliefs is by looking at the variance. We formalize a learning objective by supposing that the public seeks a publication rule that minimizes the expected variance of the posterior beliefs $\pi_{1}$. Formally, under the learning objective we replace the earlier relevance welfare function $W(D, a, \theta)$ from (1) with

$$
W\left(D, \pi_{1}\right)=-\operatorname{Var}_{\theta \sim \pi_{1}}[\theta]-D c,
$$

where $c>0$ continues to represent the social opportunity cost of publication. The learning-optimal publication rule $p$ is the one which maximizes the ex ante expectation of 14 .

When considering learning, we restrict to Bayesian updating by the public. Therefore the subjective distribution of beliefs is consistent with the distribution under the true model, and the public's subjective variance is indeed accurate given its information.

There is a clear connection between learning and relevance: under Bayesian updating, the subjective expectation of the quadratic loss relevance utility is minus the posterior variance, which is the learning welfare. So the learning-optimal policy is exactly the policy that maximizes the quadratic

loss relevance objective under Bayesian updating, as in Section 3.2, regardless of assumptions about signals or priors. In order to maximize learning and minimize uncertainty over the state of the world, then, it remains optimal to publish only those studies which induce extreme posteriors. This gives an alternative interpretation of the previous results that were motivated by decision problems. 


\subsubsection{An Accuracy Objective}

When there are normal signals, we naturally interpret the finding $X$ as a point estimate of the state $\theta$. Under an accuracy objective, a journal seeks to publish point estimates that are as close as possible to the true state of the world. These estimates can be thought of as the ones that would be the most "replicable" by future studies. Letting $\Theta=\mathcal{X}=\mathbb{R}$, we formalize our accuracy objective by replacing welfare from (1) with

$$
W(D, \theta, X)=D \cdot\left(-(X-\theta)^{2}+b\right)
$$

where $b>0$ indicates the shadow benefit of publication; if no study arrives, welfare is normalized to zero. For simplicity, we assume a quadratic loss from publishing values of $X$ further from $\theta$. (We consider a generalized loss function below.)

If the goal is to publish accurate or replicable results, a non-selective rule will do better than publishing only extreme values - but a different selective rule can do even better. Let the accuracyoptimal publication rule be the one maximizing the ex ante expectation of this welfare function.

Under the accuracy objective, publication depends only on the belief $\pi_{1}^{(X, S)}$. The accuracyoptimal rule publishes a study $(X, S)=(x, s)$ if the interim expected welfare from 15 is greater than 0 , i.e., if

$$
\mathbb{E}_{\theta \sim \pi_{1}^{(x, s)}}\left[(x-\theta)^{2}\right] \leq b .
$$

We can explicitly solve for this rule when there are normal signals and normal priors: publish if $\left(X-\mu_{0}\right)^{2} \leq\left(1+\frac{\sigma_{0}^{2}}{S^{2}}\right)\left(b+b \frac{\sigma_{0}^{2}}{S^{2}}-\sigma_{0}^{2}\right){ }^{22}$ At any standard error $S$, it is accuracy-optimal to publish studies with the point estimate $X$ in a symmetric interval about $\mu_{0}$; see Figure 6. (At sufficiently high standard errors, it may be the case that no studies are published.)

In other words, the accuracy-optimal publication rule has the opposite form of the publication rule maximizing quadratic loss relevance: at a given standard error, it publishes moderate findings and does not publish extreme ones. By the same token, publishing only extreme findings at a given standard error would minimize accuracy. This is because point estimates closer to the prior mean are thought (under the interim belief) to be closer to the true state. For intuition, recall that the distance of the point estimate from the interim mean, $X-\mu_{1}^{(X, S)}$, is linear in the distance of the point estimate from the prior mean, $X-\mu_{0}$. Of course, the accuracy-optimal publication rule is still partially aligned with the earlier (relevance-)optimal rules in that it publishes a larger range of point estimates when standard errors are smaller.

Just as the relevance-optimal rule is bad for accuracy, so too is the accuracy-optimal rule bad for relevance. For a fixed standard error and for a fixed share of studies to be published, the rule of publishing only moderate point estimates would actually minimize quadratic loss utility - and would therefore also be the worst for the learning objective ${ }^{23}$ A non-selective publication rule would be

\footnotetext{
${ }^{22}$ As a first step, one can rewrite $[16]$ as $\operatorname{Var}_{\pi_{1}^{(x, s)}}[\theta]+\left(x-\mathbb{E}_{\pi_{1}^{(x, s)}}[\theta]\right)^{2} \leq b$. We then plug in the variance and expectation from (3) to derive the publication rule above.

${ }^{23}$ As described in Section 3.1 we solved for the rule that maximized quadratic loss utility for Bayesian updating by first showing that the problem was equivalent to $\max _{p} \max _{a^{0}} E W\left(p, a^{0}\right)$; rearranging the order of maximization
} 
Figure 6: Accuracy-optimal publication region (shaded) for quadratic distance, normal prior, normal signals.

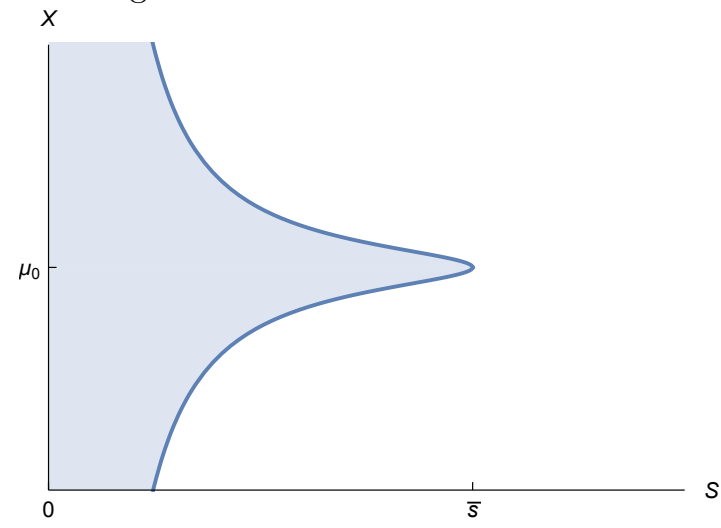

(a) In terms of the point estimate $X$, as a function of standard error $S$.

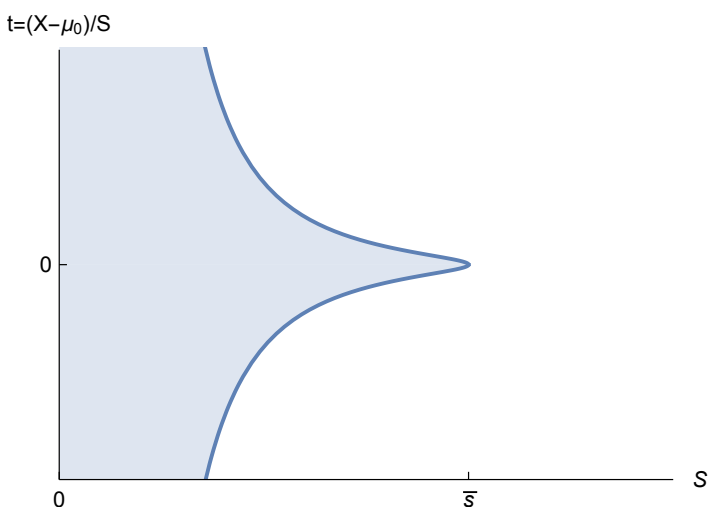

(b) In terms of the t-statistic $\left(X-\mu_{0}\right) / S$, as a function of standard error $S$.

If $b<\sigma_{0}^{2}$, as pictured, then no studies are published for $S>\bar{s}$, with $\bar{s}=\frac{\sigma_{0} \sqrt{b}}{\sqrt{\sigma_{0}^{2}-b}}$. If instead $b \geq \sigma_{0}^{2}$, then an interval of $X$ containing $\left[\mu_{0}-\left(b-\sigma_{0}^{2}\right), \mu_{0}+\left(b-\sigma_{0}^{2}\right)\right]$ would be published for any $S$.

intermediate on both quadratic loss relevance and on accuracy.

Without giving an explicit characterization, the same qualitative result of publishing moderate results to maximize accuracy would hold if we were to generalize the accuracy objective (15) beyond a quadratic cost of distance. Consider a generalized accuracy objective of

$$
W(D, \theta, X)=D \cdot\left(-\delta\left((X-\theta)^{2}\right)+b\right),
$$

for a strictly increasing function $\delta(\cdot)$. (An arbitrary increasing function of $(X-\theta)^{2}$ is equivalent to an arbitrary increasing function of $|X-\theta|$.) One can establish that under normal signals and normal priors, the generalized accuracy-optimal policy maximizing $[15]$ takes the same form as with a quadratic cost: at a given standard error, either point estimates in a symmetric interval around $\mu_{0}$ are published, or no point estimates are published. See Appendix A.3.

\section{References}

Abadie, A. (2018). On statistical non-significance in empirical economics. arXiv preprint arXiv:1803.00609.

Akerlof, G. and Michaillat, P. (2017). Beetles: Biased promotions and persistence of false belief.

Andrews, I. and Kasy, M. (2017). Identification of and correction for publication bias. NBER working paper, (23298).

let us conclude that the globally optimal $p$ was also interim-optimal given $a^{0}$. To solve for the policy that minimizes quadratic loss utility (at a fixed and commonly known standard error), one solves $\min _{p} \max _{a^{0}} E W\left(p, a^{0}\right)$. By a minimax theorem, one can rearrange the order of minimization and maximization and conclude that the globally pessimal $p$ is also interim-pessimal given $a^{0}$, and the interim-pessimal policy is to publish moderate results. 
Blackwell, D. (1953). Equivalent comparisons of experiments. The annals of mathematical statistics, pages $265-272$.

Brodeur, A., Lé, M., Sangnier, M., and Zylberberg, Y. (2016). Star wars: The empirics strike back. American Economic Journal: Applied Economics, 8(1):1-32.

Camerer, C. F., Dreber, A., Forsell, E., Ho, T.-H., Huber, J., Johannesson, M., Kirchler, M., Almenberg, J., Altmejd, A., and Chan, T. (2016). Evaluating replicability of laboratory experiments in economics. Science, 351(6280):1433-1436.

Card, D. and DellaVigna, S. (2013). Nine facts about top journals in economics. Journal of Economic Literature, 51(1):144-61.

Chambers, C., Feredoes, E., Muthukumaraswamy, S., and Etchells, P. (2014). Instead of "playing the game" it is time to change the rules: Registered reports at aims neuroscience and beyond. AIMS Neuroscience, 1(1):4-17.

Christensen, G. S. and Miguel, E. (2016). Transparency, reproducibility, and the credibility of economics research. NBER Working Paper No. 22989.

Dawid, A. P. (1973). Posterior expectations for large observations. Biometrika, 60(3):664-667.

Enke, B. (2017). What you see is all there is. Working paper.

Franco, A., Malhotra, N., and Simonovits, G. (2014). Publication bias in the social sciences: Unlocking the file drawer. Science, 345(6203):1502-1505.

Frankel, A. and Kamenica, E. (2018). Quantifying information and uncertainty. Working paper.

Furukawa, C. (2018). "unbiased" publication bias: theory and evidence. Working paper.

Gelman, A. and Loken, E. (2014). The statistical crisis in science. American Scientist, 102(6):460.

Glaeser, E. L. (2006). Researcher incentives and empirical methods.

Harbaugh, R., Maxwell, J., and Shue, K. (2016). Consistent good news and inconsistent bad news. Working paper.

Henry, E. and Ottaviani, M. (2017). Research and the approval process: the organization of persuasion.

Ioannidis, J. P. (2008). Why most discovered true associations are inflated. Epidemiology, 19(5):640648.

Ioannidis, J. P. A. (2005). Why most published research findings are false. PLoS Med, 2(8).

Lehmann, E. (1988). Comparing location experiments. The Annals of Statistics, pages 521-533.

Lehmann, E. L. and Casella, G. (1998). Theory of point estimation, volume 31. Springer.

Libgober, J. (2015). False positives in scientific research. Working Paper, Harvard University.

McClellan, A. (2017). Experimentation and approval mechanisms.

McElreath, R. and Smaldino, P. E. (2015). Replication, communication, and the population dynamics of scientific discovery. PLoS One, 10(8):e0136088.

Müller-Itten, M. (2017). Gatekeeping under asymmetric information. Working paper. 
Nissen, S. B., Magidson, T., Gross, K., and Bergstrom, C. T. (2016). Publication bias and the canonization of false facts. Elife, 5:e21451.

Nosek, B. and Lakens, D. (2014). Registered reports: a method to increase the credibility of published results. Social Psychology, 45(3):137-141.

O'Hagan, A. (1979). On outlier rejection phenomena in bayes inference. Journal of the Royal Statistical Society. Series B (Methodological), pages 358-367.

Open Science Collaboration (2015). Estimating the reproducibility of psychological science. Science, 349(6251):aac4716.

Persico, N. (2000). Information acquisition in auctions. Econometrica, 68(1):135-148.

Rosenthal, R. (1979). The file drawer problem and tolerance for null results. Psychological bulletin, 86(3):638.

Simmons, J. P., Nelson, L. D., and Simonsohn, U. (2011). False-positive psychology: Undisclosed flexibility in data collection and analysis allows presenting anything as significant. Psychological science, 22(11):1359-1366.

Sterling, T. D. (1959). Publication decisions and their possible effects on inferences drawn from tests of significance - or vice versa. Journal of the American statistical association, 54(285):30-34.

Subramanyam, K. (1996). Uncertain precision and price reactions to information. Accounting Review, pages $207-219$.

Sun, Y., Baricz, Á., and Zhou, S. (2010). On the monotonicity, log-concavity, and tight bounds of the generalized marcum and nuttall $q$-functions. IEEE Transactions on Information Theory, $56(3): 1166-1186$.

Tetenov, A. (2016). An economic theory of statistical testing. Technical report, cemmap working paper, Centre for Microdata Methods and Practice.

Van der Vaart, A. W. (2000). Asymptotic statistics (Cambridge series in statistical and probabilistic mathematics). Cambridge University Press.

Verrecchia, R. E. (1983). Discretionary disclosure. Journal of accounting and economics, 5:179-194.

Yoder, N. (2018). Designing incentives for heterogeneous researchers. Working paper. 


\section{Appendix}

\section{A Additional Results}

\section{A.1 Optimal publication without supermodularity and FOSD}

Proposition 4 argues that extreme results should be the ones to be published, assuming that the utility function is supermodular. Extreme results are defined in relation to the FOSD ordering of the induced beliefs. In this subsection we demonstrate the importance of the two conditions of supermodularity and FOSD ordering. First, we provide a simple example to show that when the utility function does not satisfy supermodularity, the publication region can consist of beliefs inside rather than outside of an interval. Second, we illustrate that even under a supermodular utility function, it is not necessarily the case that beliefs inducing more extreme actions are more valuable to publish when the beliefs are not ordered by FOSD.

\section{A.1.1 Without supermodularity}

Let $\mathcal{A}=\{0,1\}$ and $\Theta=\mathbb{R}$. Let the utility function be given by $U(0, \theta)=0$ and $U(1, \theta)=k-\theta^{2}$ for some constant $k>0{ }^{24}$ This utility function is not supermodular, as action 0 is optimal at (known) states $\theta$ for which $\theta \leq-\sqrt{k}$ or $\theta \geq \sqrt{k}$ while action 1 is optimal at states $\theta$ in $[-\sqrt{k}, \sqrt{k}]$.

Assume that there is a normal prior and normal signals, so that the interim belief after observing a signal $X$ is given by $\pi_{1}^{(X, S)}=\mathcal{N}\left(\mu_{1}, \sigma_{1}^{2}\right)$, with

$$
\begin{aligned}
\mu_{1} & =\frac{\sigma_{0}^{2}}{S^{2}+\sigma_{0}^{2}} X+\frac{S^{2}}{S^{2}+\sigma_{0}^{2}} \mu_{0} \\
\sigma_{1}^{2} & =\frac{S^{2} \sigma_{0}^{2}}{S^{2}+\sigma_{0}^{2}} .
\end{aligned}
$$

At any given standard error $S=s$, these interim beliefs are FOSD ordered in $X$. The expected utility of action $a=1$ after observing study $(X, S)$ is therefore given by $\mathbb{E}_{\theta \sim \pi_{1}^{(X, S)}}\left[k-\theta^{2}\right]=k-\mu_{1}^{2}-\sigma_{1}^{2}$. Action $a=1$ would be taken if $\mu_{1}^{2}+\sigma_{1}^{2}<k$, and action $a=0$ would be taken if $\mu_{1}^{2}+\sigma_{1}^{2}>k$.

So, if the default action is $a^{0}=0$, take some $S=s$ such that $\sigma_{1}^{2}=\frac{s^{2} \sigma_{0}^{2}}{s^{2}+\sigma_{0}^{2}}<k$. Then the gross interim benefit of publishing a study $(X, s)$ will be

$$
\Delta\left(\pi_{1}^{(X, s)}, 0\right)=\max \left\{k-\left(\frac{\sigma_{0}^{2}}{s^{2}+\sigma_{0}^{2}} X+\frac{s^{2}}{s^{2}+\sigma_{0}^{2}} \mu_{0}\right)^{2}-\frac{s^{2} \sigma_{0}^{2}}{s^{2}+\sigma_{0}^{2}}, 0\right\}
$$

For a low enough cost $c$ that the journal would publish any result at $S=s$, the interim optimal publication rule would be to publish only those values of $X$ inside of a bounded interval.

Moving beyond this example, suppose that we maintain the assumption of normal priors and signals, and we fix a standard error. Given an essentially arbitrary union of disconnected intervals of point estimates, one could motivate that set as the interim optimal publication region by defining an appropriate utility function.

\section{A.1.2 Supermodularity without FOSD}

One might conjecture that as long as the utility function satisfies supermodularity, then the conclusion of Proposition 4 applies not just to beliefs $\pi^{\prime}, \pi^{\prime \prime}, \pi^{\prime \prime \prime}$ ordered by FOSD, but to beliefs that are ordered by the induced actions $a^{*}(\pi)$. The following example provides a counterexample.

\footnotetext{
${ }^{24}$ Note that while the action space here is binary, this utility function differs from the "binary action" utility. That utility function was without loss of generality on the binary action space only until one adds assumptions on priors and signal distributions.
} 
Let the state space and action space both be given by $\mathcal{A}=\Theta=\{0,1,2\}$. Define the utility function $U$ by

$$
U(a, \theta)=\begin{array}{lc|ccc} 
& & \multicolumn{4}{|c}{\theta} \\
a & 0 & 0 & 1 & 2 \\
\cline { 2 - 5 } & 0 & -10 & 10 & 11 \\
& 2 & -15 & 6 & 20
\end{array}
$$

This utility function is supermodular. Higher beliefs lead to higher actions, and if the state is known to be $\theta$ with certainty, then the corresponding optimal action is $a=\theta$.

Next, let $a^{0}=0$; let $\pi^{\prime}$ be state $\theta=0$ with certainty; let $\pi^{\prime \prime}$ be state $\theta=1$ with certainty; and let $\pi^{\prime \prime \prime}$ be a $50 \%$ probability of $\theta=0$ and a $50 \%$ probability of $\theta=2$. It holds that $a^{*}\left(\pi^{\prime}\right)=$ $0, a^{*}\left(\pi^{\prime \prime}\right)=1$, and $a^{*}\left(\pi^{\prime \prime \prime}\right)=2$, but these beliefs are not ordered by FOSD. The gross interim benefits of publishing studies leading to these beliefs are given by $\Delta\left(\pi^{\prime}, 0\right)=0, \Delta\left(\pi^{\prime \prime}, 0\right)=10$, and $\Delta\left(\pi^{\prime \prime \prime}, 0\right)=2.5$. Hence, it is most valuable to publish the study leading to the belief $\pi^{\prime \prime}$ that induces the intermediate action.

\section{A.2 Size control for selective publication rules}

Let there be normal signals, and fix $z>0$. In this subsection we show how to construct selective publication rules for which the coverage probability of the confidence interval $[X-z S, X+z S]$ is equal to $\Phi[z]-\Phi[-z]$ for all $\theta$. This exercise demonstrates that while non-selectivity is sufficient for confidence intervals (at a fixed standard error radius about the point estimate) to control size, it is not necessary.

Case of $\mathbf{S}=1$ : Normalizing $S=1$, let the distribution of the finding $X$ be given by $X \sim N(\theta, 1)$ and the publication probability be given by $p(X)$. Then the coverage probability of a confidence interval of the form $[X-z, X+z]$ is given by

$$
P(\theta \in[X-z, X+z])=\frac{\int p(\theta+\epsilon) \mathbf{1}(\epsilon \in[-z, z]) \varphi(\epsilon) d \epsilon}{\int p(\theta+\epsilon) \varphi(\epsilon) d \epsilon} .
$$

This coverage probability is equal to its nominal level, $\Phi(z)-\Phi(-z)$, for all $\theta$, if and only if

$$
\int p(\theta+\epsilon)[\mathbf{1}(\epsilon \in[-z, z])-(\Phi(z)-\Phi(-z))] \varphi(\epsilon) d \epsilon=0 \text { for all } \theta .
$$

Taking the Fourier transform $\mathscr{F}$ of this expression, and recalling that the Fourier transform maps convolutions into products, the above expression is equivalent to the condition

$$
\mathscr{F}(p(\cdot)) \cdot \mathscr{F}([\mathbf{1}(\cdot \in[-z, z])-(\Phi(z)-\Phi(-z))] \varphi(\cdot)) \equiv 0 .
$$

If the coverage probability is equal to its nominal level, we thus get that $\mathscr{F}(p(\cdot))$ has to equal zero everywhere except possibly at points where $\mathscr{F}([\mathbf{1}(\cdot \in[-z, z])-(\Phi(z)-\Phi(-z))] \varphi(\cdot))=0$. Reversely, by the Fourier inversion theorem 25 this condition is also sufficient for the coverage probability to be equal to its nominal level.

The Fourier transform $\mathscr{F}([\mathbf{1}(\cdot \in[-z, z])-(\Phi(z)-\Phi(-z))] \varphi(\cdot))$ is real-valued, even, and continuous. Let $t^{*}$ be any zero of this Fourier transform. Then for any publication rule of the form $p(x)=r_{0}+r_{1} \cdot \sin \left(t^{*} \cdot x\right)+r_{2} \cdot \cos \left(t^{*} \cdot x\right)$ we get that nominal size control is satisfied. (Of course, one must ensure that the publication probability is bounded between 0 and 1.) We can also take

25 https://en.wikipedia.org/wiki/Fourier_inversion_theorem 
linear combinations of these functions over different roots $t^{*}$. These are the only publication rules with nominal size control.

While we cannot obtain analytic solutions, at any $z$ we can numerically solve for such roots. For instance, for $z=1.96$, solutions include $t^{*} \simeq 2.11045,3.49544$, etc. So under either of the publication rules $p(x)=.5+.5 \cos (2.11045 x)$ or $p(x)=.5+.5 \cos (3.49544 x)$, for example, the probability of $\theta \in[X-1.96, X+1.96]$ conditional on publication would be $95 \%$ at all $\theta$.

General case: Fixing $z$, suppose that $p(x)$ is some publication rule that satisfies nominal coverage for $S=1$. Then $p(x, s)=p(x / s)$ achieves nominal coverage for $S=s$.

\section{A.3 Generalized accuracy objectives}

The generalized accuracy objective was defined as

$$
W(D, \theta, X)=D \cdot\left(-\delta\left((X-\theta)^{2}\right)+b\right)
$$

for $\delta$ some increasing function. To maximize this objective under normal priors and normal signals, it is optimal to publish studies with moderate results, i.e., ones with point estimates $X$ inside of an interval centered at the prior mean $\mu_{0}$ :

Proposition 11. Let there be normal priors and normal signals. The publication rule maximizing the generalized accuracy objective (15] takes the following form: at $S=s$, either no studies $(X, s)$ are published, or there exists $k$ such that a study $(X, s)$ is published if and only if $\left(X-\mu_{0}\right)^{2} \leq k$.

\section{B Proofs}

Proof of Lemma 1. Follows from arguments in the text.

Proof of Lemma 2. Follows from arguments in the text.

Proof of Proposition 1. Follows from arguments in the text.

Proof of Proposition 2. By Proposition 1 part 2a it suffices to show that $a=a^{*}\left(\pi_{1}^{0, p^{I(a)}}\right)$ is uniquely solved by $a=\mu_{0}$ - in other words, that $a^{0}=\mu_{0}$ is the unique fixed point when we map default actions to interim optimal publication rules, and then map publication rules back to default actions.

Conditional on a study arriving when the default action is $a^{0}$, the journal will not publish a study if $\mu(X, S)$ lies in the interval $\left(a^{0}-\sqrt{c}, a^{0}+\sqrt{c}\right)$ (see Equation 8). Let $\bar{\mu}\left(a^{0}\right)$ indicate $\mathbb{E}\left[\theta \mid \mu_{1}^{(X, S)} \in\left(a^{0}-\sqrt{c}, a^{0}+\sqrt{c}\right)\right]$, the expected state conditional on a study arriving and not being published. If this expectation is undefined due to the event $\mu_{1}^{(X, S)} \in\left(a^{0}-\sqrt{c}, a^{0}+\sqrt{c}\right)$ occurring with zero probability, let $\bar{\mu}\left(a^{0}\right)=\mu_{0}$.

The mean of the default belief - and therefore the implied default action - conditional on nonpublication will be a convex combination of $\bar{\mu}\left(a^{0}\right)$ (with weight $q$ ) and $\mu_{0}$ (weight $1-q$ ). Therefore, to show that $a^{0}=\mu_{0}$ is the unique fixed point, it is sufficient to show the following three items: (i) for $a^{0}=\mu_{0}$, it holds that $\bar{\mu}\left(a^{0}\right)=a^{0}$; (ii) for any $a^{0}<\mu_{0}$, it holds that $\bar{\mu}\left(a^{0}\right)>a^{0}$; and (iii) for any $a^{0}>\mu_{0}$, it holds that $\bar{\mu}\left(a^{0}\right)<a^{0}$. (If we had assumed $q<1$ then it would be sufficient to show (ii) and (iii) with weak inequalities.)

Item (i) follows from the fact that $\mu_{1}^{(X, S)}$ is symmetric about $\mu_{0}$, and therefore it remains symmetric when this random variable is truncated outside of the interval $\left(\mu_{0}-\sqrt{c}, \mu_{0}+\sqrt{c}\right)$. The proofs of items (ii) and (iii) will be identical to each other, up to the direction of inequalities, so let us focus on proving (ii). Fix $a^{0}<\mu_{0}$. First, if there is a zero probability that $\mu_{1}^{(X, S)} \in\left(a^{0}-\sqrt{c}, a^{0}+\sqrt{c}\right)$, then $\bar{\mu}\left(a^{0}\right)=\mu_{0}>a^{0}$ and we are done. Otherwise, notice that symmetry about $\mu_{0}$ combined with 
single-peakedness means that the pdf of $\mu_{1}^{(X, S)}$ is larger at $a^{0}+k$ than at $a^{0}-k$ for any $k>0$, with the inequality being strict for any $\epsilon$ such that either pdf value is nonzero. Hence the mean of $\mu_{1}^{(X, S)}$ conditional on being in the interval $\left(a^{0}-\sqrt{c}, a^{0}+\sqrt{c}\right)$ is strictly above the midpoint $a^{0}$. That completes the proof of item (ii).

Proof of Corollary 1. As discussed in the text, this prior and signal structure satisfy the hypotheses of Proposition 2, and so the optimal rule is to publish if $\left|\mu_{1}^{(X, S)}-\mu_{0}\right| \geq \sqrt{c}$. By the normal updating formula (3), $\mu_{1}^{(X, S)}=\frac{\sigma_{0}^{2}}{S^{2}+\sigma_{0}^{2}} X+\frac{S^{2}}{S^{2}+\sigma_{0}^{2}} \mu_{0}$, and so $\left|\mu_{1}^{(X, S)}-\mu_{0}\right|=\frac{\sigma_{0}^{2}}{S^{2}+\sigma_{0}^{2}}\left|X-\mu_{0}\right|=$ $\left(1+\frac{S^{2}}{\sigma_{0}^{2}}\right)^{-1}\left|X-\mu_{0}\right|$.

Proof of Corollary 2. The only comparative static that is not immediate is that for the t-statistic cutoff, $\left(\frac{1}{S}+\frac{S}{\sigma_{0}^{2}}\right) \sqrt{c}$, with respect to $S$. Taking straightforward limits confirms that the cutoff goes to infinity as $S \rightarrow 0$ and $S \rightarrow \infty$. The derivative of the cutoff with respect to $S$ is $\left(-\frac{1}{S^{2}}+\frac{1}{\sigma_{0}^{2}}\right) \sqrt{c}$, and the second derivative is $\frac{2 \sqrt{c}}{S^{3}}$. Since the second derivative is positive, the cutoff is convex over $S \in \mathbb{R}_{++}$and is minimized at the point where the first derivative is 0 , which is $S=\sigma_{0}^{2}$.

Proof of Proposition 3. By Proposition 1 part 2b, it suffices to show that the payoff under default action $a^{0}=0$ is higher than under default action $a^{0}=1$, i.e., that $\operatorname{EW}\left(p^{I(0)}, 0\right) \geq E W\left(p^{I(1)}, 1\right)$. The interim optimal publication rule $p^{I\left(a^{0}\right)}$ is given by (9): for $a^{0}=0$, publish if $\mu_{1}^{(X, S)} \geq c$, and for $a^{0}=1$, publish if $\mu_{1}^{(X, S)} \leq-c$. Expanding out $E W\left(p, a^{0}\right)$ from (4) for each possible value of $a^{0}$,

$$
\begin{aligned}
& E W\left(p^{I(0)}, 0\right)=q \mathbb{E}\left[\begin{array}{ll}
\mu_{1}^{(X, S)}-c & \text { if } \mu_{1}^{(X, S)} \geq c \\
0 & \text { if } \mu_{1}^{(X, S)}<c
\end{array}\right] \\
& E W\left(p^{I(1)}, 1\right)=q \mathbb{E}\left[\left\{\begin{array}{ll}
\mu_{1}^{(X, S)} & \text { if } \mu_{1}^{(X, S)}>-c \\
-c & \text { if } \mu_{1}^{(X, S)} \leq-c
\end{array}\right]+(1-q) \mu_{0} .\right.
\end{aligned}
$$

Taking the difference,

$$
E W\left(p^{I(0)}, 0\right)-E W\left(p^{I(1)}, 1\right)=q \mathbb{E}\left[\left\{\begin{array}{ll}
-c & \text { if } \mu_{1}^{(X, S)} \geq c \\
-\mu_{1}^{(X, S)} & \text { if } \mu_{1}^{(X, S)} \in(-c, c) \\
c & \text { if } \mu_{1}^{(X, S)} \leq-c
\end{array}\right]-(1-q) \mu_{0} .\right.
$$

We seek to show that this difference is nonnegative. Since $\mu_{0} \leq 0$ by assumption, it is sufficient to show that the expectation term is nonnegative.

To show that the expectation term is nonnegative, first define a weakly increasing function $l: \mathbb{R} \rightarrow \mathbb{R}_{+}$as follows:

$$
l(k)= \begin{cases}0 & \text { if } k \leq 0 \\ k & \text { if } k \in(0, c) . \\ c & \text { if } k>c\end{cases}
$$

The expectation term in 17 can be rewritten as $\mathbb{E}\left[l\left(-\mu_{1}^{(X, S)}\right)\right]-\mathbb{E}\left[l\left(\mu_{1}^{(X, S)}\right)\right]$, and so it is sufficient to show that this difference is nonnegative. 
Next, observe that the distribution of $-\mu_{1}^{(X, S)}$ first order stochastically dominates that of $\mu_{1}^{(X, S)}$ :

$$
\begin{aligned}
P\left(-\mu_{1}^{(X, S)} \leq k\right) & =1-P\left(\mu_{1}^{(X, S)} \leq-k\right) \\
& \leq 1-P\left(\mu_{1}^{(X, S)} \geq k\right) \\
& =P\left(\mu_{1}^{(X, S)} \leq k\right),
\end{aligned}
$$

where the inequality comes from the assumption of $P\left(\mu_{1}^{(X, S)} \leq-k\right) \geq P\left(\mu_{1}^{(X, S)} \geq k\right)$. By FOSD, then, the expectation of $l\left(-\mu_{1}^{(X, S)}\right)$ is weakly larger than the expectation of $l\left(\mu_{1}^{(X, S)}\right)$, completing the proof.

Proof of Corollary 3. As discussed in the text, this prior and signal structure satisfy the hypotheses of Proposition 3, and so the optimal rule is to publish if $\mu_{1}^{(X, S)} \geq c$. By the normal updating formula (3), $\mu_{1}^{(X, S)}=\frac{\sigma_{0}^{2}}{\S^{2}+\sigma_{0}^{2}} X+\frac{S^{2}}{S^{2}+\sigma_{0}^{2}} \mu_{0}$. Rearranging, $\frac{\sigma_{0}^{2}}{\S^{2}+\sigma_{0}^{2}} X+\frac{S^{2}}{S^{2}+\sigma_{0}^{2}} \mu_{0} \geq c$ if and only if $X \geq\left(1+\frac{S^{2}}{\sigma_{0}^{2}}\right) c-\frac{S^{2}}{\sigma_{0}^{2}} \mu_{0}$.

Proof of Corollary 4. The only comparative static that is not immediate is that for the t-statistic cutoff, $\left(\frac{1}{S}+\frac{S}{\sigma_{0}^{2}}\right)\left(c-\mu_{0}\right)$, with respect to $S$. The argument for this result follows identically as the argument for the analogous result in the proof of Corollary 2.

Proof of Proposition 4. Let $a^{\prime}=a^{*}\left(\pi^{\prime}\right), a^{\prime \prime}=a^{*}\left(\pi^{\prime \prime}\right)$, and $a^{\prime \prime \prime}=a^{*}\left(\pi^{\prime \prime \prime}\right)$. Moreover, recall that for any actions $\underline{a} \leq \bar{a}$ and any distributions $\underline{\pi} \leq_{F O S D} \bar{\pi}$, supermodularity implies that

$$
\mathbb{E}_{\theta \sim \bar{\pi}}[U(\underline{a}, \theta)]+\mathbb{E}_{\theta \sim \underline{\pi}}[U(\bar{a}, \theta)] \leq \mathbb{E}_{\theta \sim \pi}[U(\underline{a}, \theta)]+\mathbb{E}_{\theta \sim \bar{\pi}}[U(\bar{a}, \theta)] .
$$

Now consider the two exhaustive cases of $a^{0} \leq a^{\prime \prime}$ and $a^{0} \geq a^{\prime \prime}$.

If $a^{0} \leq a^{\prime \prime}$, then

$$
\begin{gathered}
\mathbb{E}_{\theta \sim \pi^{\prime \prime \prime}}\left[U\left(a^{0}, \theta\right)\right]+\mathbb{E}_{\theta \sim \pi^{\prime \prime}}\left[U\left(a^{\prime \prime}, \theta\right)\right] \leq \mathbb{E}_{\theta \sim \pi^{\prime \prime}}\left[U\left(a^{0}, \theta\right)\right]+\mathbb{E}_{\theta \sim \pi^{\prime \prime \prime}}\left[U\left(a^{\prime \prime}, \theta\right)\right] \\
\leq \mathbb{E}_{\theta \sim \pi^{\prime \prime}}\left[U\left(a^{0}, \theta\right)\right]+\mathbb{E}_{\theta \sim \pi^{\prime \prime \prime}}\left[U\left(a^{\prime \prime \prime}, \theta\right)\right] \\
\Rightarrow \mathbb{E}_{\theta \sim \pi^{\prime \prime}}\left[U\left(a^{\prime \prime}, \theta\right)\right]-\mathbb{E}_{\theta \sim \pi^{\prime \prime}}\left[U\left(a^{0}, \theta\right)\right] \leq \mathbb{E}_{\theta \sim \pi^{\prime \prime \prime}}\left[U\left(a^{\prime \prime \prime}, \theta\right)\right]-\mathbb{E}_{\theta \sim \pi^{\prime \prime \prime}}\left[U\left(a^{0}, \theta\right)\right] \\
\Rightarrow \Delta\left(\pi^{\prime \prime}, a^{0}\right) \leq \Delta\left(\pi^{\prime \prime \prime}, a^{0}\right),
\end{gathered}
$$

where, on the first line, the first inequality follows from 180 and the second inequality follows from the fact that $a^{\prime \prime \prime}=a^{*}\left(\pi^{\prime \prime \prime}\right)$. The second line then rearranges terms from the left-hand side and the right-hand side of the first line.

Alternatively, if $a^{0} \geq a^{\prime \prime}$, then by a similar argument

$$
\begin{aligned}
& \mathbb{E}_{\theta \sim \pi^{\prime}}\left[U\left(a^{0}, \theta\right)\right]+\mathbb{E}_{\theta \sim \pi^{\prime \prime}}\left[U\left(a^{\prime \prime}, \theta\right)\right] \leq \mathbb{E}_{\theta \sim \pi^{\prime \prime}}\left[U\left(a^{0}, \theta\right)\right]+\mathbb{E}_{\theta \sim \pi^{\prime}}\left[U\left(a^{\prime \prime}, \theta\right)\right] \\
& \leq \mathbb{E}_{\theta \sim \pi^{\prime \prime}}\left[U\left(a^{0}, \theta\right)\right]+\mathbb{E}_{\theta \sim \pi^{\prime}}\left[U\left(a^{\prime}, \theta\right)\right] \\
& \Rightarrow \mathbb{E}_{\theta \sim \pi^{\prime \prime}}\left[U\left(a^{\prime \prime}, \theta\right)\right]-\mathbb{E}_{\theta \sim \pi^{\prime \prime}}\left[U\left(a^{0}, \theta\right)\right] \leq \mathbb{E}_{\theta \sim \pi^{\prime}}\left[U\left(a^{\prime}, \theta\right)\right]-\mathbb{E}_{\theta \sim \pi^{\prime}}\left[U\left(a^{0}, \theta\right)\right] \\
& \Rightarrow \Delta\left(\pi^{\prime \prime}, a^{0}\right) \leq \Delta\left(\pi^{\prime}, a^{0}\right) .
\end{aligned}
$$

Proof of Corollary 5. Follows from arguments in the text.

Proof of Lemma 3. As stated, when publication is non-selective, the distribution of $X \mid \theta, S=s, D=$ 1 is identical to the distribution $X \mid \theta, S=s$ for every $s$. Parts 1 and 2 follow immediately from that observation. Part 3 follows from the definition of non-selective publication: $p(x, s)$ constant in $x$ implies that $\mathbb{E}[p(X, S) \mid \theta, S=s]$ is equal to that same constant. To show part 4 , recall that 
the independence of $S$ and $\theta$ implies that if $\mathbb{E}[p(X, S) \mid \theta, S=s]$ is constant for each $s$, then it is constant in expectation across $S$, and so $\mathbb{E}[p(X, S) \mid \theta]$ is constant as well. The result then follows from (11).

Proof of Proposition 5 .

- Part $1 \Rightarrow$ all other parts: Non-selectivity of part 1 implies the other parts by Lemma 3 . Specifically, part $1 \Rightarrow$ part 2 by part 1 of Lemma 3 (plugging in $\widehat{g}(X, S)=X$ and $g(\theta, S)=\theta$ ); part $1 \Rightarrow$ part 3 by part 3 of Lemma 3 and part $1 \Rightarrow$ part 4 by part 4 of Lemma 3 .

- Part $3 \Rightarrow$ part 1. Fixing $S=s$, recall that $X$ is a complete statistic for $\theta$ in the normal location model when $\Theta_{0}$ contains an open set in $\mathbb{R}$; see for instance Theorem 6.22 in Lehmann and Casella (1998). Completeness means that for any measurable function $g: \mathcal{X} \rightarrow \mathbb{R}$, if $\mathbb{E}[g(X) \mid \theta, S=s]=0$ for all $\theta \in \Theta_{0}$, then $P(g(X)=0 \mid \theta, S=s)=1$ for all $\theta \in \Theta_{0}$. Apply this definition to $g(x)=p(x, s)-\mathbb{E}[p(X, s) \mid S=s]$. Assuming part 3 , that the publication probability is constant over $\theta \in \Theta_{0}$, it holds that the expectation of $g(X)$ is 0 for all $\theta \in \Theta_{0}$, and hence that $p(X, s)=\mathbb{E}[p(X, s) \mid S=s]$ with probability 1 given $\theta$ and $S=s$, establishing part 1

- Part 2 $\Rightarrow$ part 3. To simplify notation, consider without loss of generality the case $s=1$. Then the unbiasedness condition $\mathbb{E}[X \mid \theta, S=1, D=1]$ can be written as

$$
\frac{\int x \varphi(x-\theta) p(x, 1) d x}{\int \varphi(x-\theta) p(x, 1) d x}=\theta .
$$

Equivalently, using the fact that $\varphi^{\prime}(x)=-x \cdot \varphi(x)$,

$$
\begin{aligned}
0 & =\int(x-\theta) \varphi(x-\theta) p(x, 1) d x \\
& =-\int \varphi^{\prime}(x-\theta) p(x, 1) d x \\
& =\partial_{\theta}\left[\int \varphi(x-\theta) p(x, 1) d x\right] \\
& =\partial_{\theta} \mathbb{E}[p(X, S) \mid \theta, S=1] .
\end{aligned}
$$

If the last line is equal to 0 then $E[p(X, S) \mid \theta, S=1]$ is constant over $\theta$ in any open set contained in the support. The same argument applies for all other values of $S$.

- Part $4 \Rightarrow$ part 3 . Restating (11), the relative density of the Bayesian default belief to the prior is given by

$$
\frac{d \pi_{1}^{0, p}}{d \pi_{0}}(\theta)=\frac{1-q \cdot \mathbb{E}[p(X, S) \mid \theta]}{1-q \cdot \mathbb{E}[p(X, S)]} .
$$

The Bayesian default belief is equal to the prior when, under the prior $\theta \sim \pi_{0}$, this relative density is almost surely constant in $\theta$ (in which case the ratio is identically equal to 1 ). In other words, it holds when $\mathbb{E}[p(X, S) \mid \theta]$ is almost surely constant in $\theta$. Moreover, note that $\mathbb{E}[p(X, S) \mid \theta]$ must be continuous in $\theta$ since the signal density function $f_{X \mid \theta, S}(x \mid \theta, s)$ is a smooth function of $\theta$ for all $x, s$. Hence, if the Bayesian default belief is equal to the prior, then $\mathbb{E}[p(X, S) \mid \theta]$ must be constant in $\theta$ over the support of the prior.

Now, highlighting the dependence of this publication probability on the distribution $F_{S}$,

$$
\mathbb{E}[p(X, S) \mid \theta]=\int_{s \in \mathcal{S}} \mathbb{E}[p(X, S) \mid \theta, S=s] d F_{S}(s) .
$$


We see that the LHS of this equation is constant over $\theta$ in the support of the prior for all distributions $F_{S}$ if and only if, for all $s, \mathbb{E}[p(X, S) \mid \theta, S=s]$ is constant over $\theta$ in the support. (If there exists $s^{\prime}$ such that $\mathbb{E}\left[p(X, S) \mid \theta, S=s^{\prime}\right]$ varies in $\theta$, then the distribution $F_{S}$ placing all probability mass on $s^{\prime}$ will have $\mathbb{E}[p(X, S) \mid \theta]$ vary in $\theta$.) So if the Bayesian default belief is equal to the prior for all $F_{S}$, then the publication probability is constant over $\theta$ in $\Theta_{0}$ for all $s$.

Proof of Proposition 6. Without loss of generality, fix $s=1$. First consider the case of a bounded interval $I(1)$. Then there exist $\theta^{\prime}$ (the midpoint of the interval) and $y>0$ (the radius) such that $I(1)=\left[\theta^{\prime}-y, \theta^{\prime}+y\right]$. If $y>z$,

$$
P\left(\theta^{\prime} \in[X-z, X+z] \mid \theta=\theta^{\prime}, S=1, D=1\right)=0,
$$

and the result follows. If $y \leq z$, applying the law of iterated expectations and letting $C=1$ denote the event of study submission,

$$
\begin{aligned}
\Phi(z)-\Phi(-z)= & P\left(\theta^{\prime} \in[X-z, X+z] \mid \theta=\theta^{\prime}, S=1, C=1\right) \\
= & P\left(D=0 \mid \theta=\theta^{\prime}, S=1, C=1\right) \cdot P\left(\theta^{\prime} \in[X-z, X+z] \mid \theta=\theta^{\prime}, S=1, C=1, D=0\right) \\
& +P\left(D=1 \mid \theta=\theta^{\prime}, S=1, C=1\right) \cdot P\left(\theta^{\prime} \in[X-z, X+z] \mid \theta=\theta^{\prime}, S=1, C=1, D=1\right) .
\end{aligned}
$$

Conditional on a study submitted but not published, it holds that $X \in\left[\theta^{\prime}-y, \theta^{\prime}+y\right]$, and therefore since $y \leq z$ that $\theta^{\prime} \in[X-z, X+z]$ :

$$
P\left(\theta^{\prime} \in[X-z, X+z] \mid \theta=\theta^{\prime}, S=1, C=1, D=0\right)=1 .
$$

Therefore $\Phi(z)-\Phi(-z)$ is equal to a weighted average of 1 and $P\left(\theta^{\prime} \in[X-z, X+z] \mid \theta=\theta^{\prime}, S=\right.$ $1, D=1, C=1)$ - with positive weights on both - and hence $P\left(\theta^{\prime} \in[X-z, X+z] \mid \theta=\theta^{\prime}, S=\right.$ $1, D=1, C=1)<\Phi(z)-\Phi(-z)$, yielding the desired result.

Consider finally the case of unbounded $I(1)$. If $I(1)=(-\infty, y]$ for some $y$, then for $\theta^{\prime}<y-z$,

$$
P\left(\theta^{\prime} \in[X-z \cdot s, X+z \cdot s] \mid \theta=\theta^{\prime}, S=s, D=1\right)=0<\Phi(z)-\Phi(-z) .
$$

A symmetric argument holds for $I(1)=[y, \infty)$ and $\theta^{\prime}>y+z$, concluding our proof.

Proof of Proposition 7 . As stated in the text, the result follows from the fact that the signal $X \mid S=s$ distributed according to $\mathcal{N}\left(\theta, s^{2}\right)$ is a Blackwell more informative signal of $\theta$ when $s$ is smaller. Blackwell more informative signals have higher expected value to a decisionmaker regardless of utility function $U$ or prior $\pi_{0}$. Moreover, as $s \rightarrow \infty$, the signal becomes uninformative and the expected benefit of publication goes to 0 , which is below $c>0$.

Proof of Proposition 8. As with the one-period problem, the optimal publication rule is the one that is interim optimal given the naive default belief $\pi_{1}^{0}=\pi_{0}$.

Suppose a study $\left(X_{1}, S_{1}\right)$ arrives at period 1 . Let $\mu_{1}^{0}$ indicate the posterior mean at period 1 in the absence of publication, and $\mu_{1}^{\left(X_{1}, S_{1}\right)}$ the posterior mean at period 1 if the study is published. Let $\mu_{2}^{0,\left(X_{2}\right)}$ indicate the posterior mean at period 2 if the study had not been published and then the second period signal is observed to be $X_{2}$, and $\mu_{2}^{\left(X_{1}, S_{1}\right),\left(X_{2}\right)}$ the posterior mean at period 2 if the study had been published. We can calculate these posterior means as follows: 


$$
\begin{aligned}
\mu_{1}^{0} & =\mu_{0} \\
\mu_{1}^{\left(X_{1}, S_{1}\right)} & =\frac{1}{\frac{1}{\sigma_{0}^{2}}+\frac{1}{S_{1}^{2}}}\left(\frac{\mu_{0}}{\sigma_{0}^{2}}+\frac{X_{1}}{S_{1}^{2}}\right) \\
\mu_{2}^{0,\left(X_{2}\right)} & =\frac{1}{\frac{1}{\sigma_{0}^{2}}+\frac{1}{s_{2}^{2}}}\left(\frac{\mu_{0}}{\sigma_{0}^{2}}+\frac{X_{2}}{s_{2}^{2}}\right) \\
\mu_{2}^{\left(X_{1}, S_{1}\right),\left(X_{2}\right)} & =\frac{1}{\frac{1}{\sigma_{0}^{2}}+\frac{1}{S_{1}^{2}}+\frac{1}{s_{2}^{2}}}\left(\frac{\mu_{0}}{\sigma_{0}^{2}}+\frac{X_{1}}{S_{1}^{2}}+\frac{X_{2}}{s_{2}^{2}}\right)
\end{aligned}
$$

Consider the interim stage, at which $\left(X_{1}, S_{1}\right)$ has been observed by the journal and not yet published, and hence at which the journal has interim belief $\pi_{1}^{1}\left(X_{1}, S_{1}\right)$. From this interim perspective, publication has a cost of $c$. It then delivers a benefit towards the first-period action payoff, and a benefit towards the second-period action payoff.

The benefit of publication towards the first-period payoff is $\alpha\left(\mu_{1}^{1}\left(X_{1}, S_{1}\right)-\mu_{1}^{0}\right)^{2}$, which simplifies to

$$
\alpha\left(\mu_{1}^{1}\left(X_{1}, S_{1}\right)-\mu_{1}^{0}\right)^{2}=\alpha \frac{\sigma_{0}^{4}}{\left(\sigma_{0}^{2}+S_{1}^{2}\right)^{2}}\left(X_{1}-\mu_{0}\right)^{2}
$$

The period 2 action if the study is published is $\mu_{2}^{1}\left(X_{1}, S_{1}, X_{2}\right)$, and is $\mu_{2}^{0}\left(X_{2}\right)$ otherwise. Hence, conditional on $X_{2}$, the benefit of first-period publication towards the second-period payoff is $(1-$ $\alpha)\left(\mu_{2}^{1}\left(X_{1}, S_{1}, X_{2}\right)-\mu_{2}^{0}\left(X_{2}\right)\right)^{2}$. At the interim stage, then, the expected second-period payoff is the expectation of that value over the random variable $X_{2}$, given beliefs $\theta \sim \pi_{1}^{1}\left(X_{1}, S_{1}\right)$ and $X_{2} \sim$ $\mathcal{N}\left(\theta, s_{2}^{2}\right)$. Writing out this expectation and simplifying,

$$
\begin{aligned}
& \mathbb{E}\left[(1-\alpha)\left(\mu_{2}^{1}\left(X_{1}, S_{1}, X_{2}\right)-\mu_{2}^{0}\left(X_{2}\right)\right)^{2} \mid X_{1}, S_{1}\right] \\
& \quad=(1-\alpha)\left(\mathbb{E}\left[\mu_{2}^{1}\left(X_{1}, S_{1}, X_{2}\right)-\mu_{2}^{0}\left(X_{2}\right) \mid X_{1}, S_{1}\right]^{2}+\operatorname{Var}\left[\mu_{2}^{1}\left(X_{1}, S_{1}, X_{2}\right)-\mu_{2}^{0}\left(X_{2}\right) \mid X_{1}, S_{1}\right]\right)
\end{aligned}
$$

Next observe that, given $X_{1}$ and $S_{1}$, the conditional distribution of $X_{2}$ is

$$
X_{2} \mid\left(X_{1}, S_{1}\right) \sim \mathcal{N}\left(\mu_{1}^{1}\left(X_{1}, S_{1}\right), \frac{1}{\frac{1}{\sigma_{0}^{2}}+\frac{1}{S_{1}^{2}}}+s_{2}^{2}\right) .
$$

Plugging this conditional distribution into the various terms of 20 and simplifying,

$$
\begin{aligned}
(1-\alpha) \mathbb{E}\left[\mu_{2}^{1}\left(X_{1}, S_{1}, X_{2}\right)-\mu_{2}^{0}\left(X_{2}\right) \mid X_{1}, S_{1}\right]^{2} & =(1-\alpha)\left(\frac{\sigma_{0}^{2} s_{2}^{2}}{\left(\sigma_{0}^{2}+S_{1}^{2}\right)\left(\sigma_{0}^{2}+s_{2}^{2}\right)}\right)^{2}\left(X_{1}-\mu_{0}\right)^{2} \\
(1-\alpha) \operatorname{Var}\left[\mu_{2}^{1}\left(X_{1}, S_{1}, X_{2}\right)-\mu_{2}^{0}\left(X_{2}\right) \mid X_{1}, S_{1}\right] & =(1-\alpha) \frac{\sigma_{0}^{8} s_{2}^{4}}{\left(\sigma_{0}^{2}+S_{1}^{2}\right)\left(\sigma_{0}^{2}+s_{2}^{2}\right)^{2}\left(\sigma_{0}^{2} S_{1}^{2}+\sigma_{0}^{2} s_{2}^{2}+S_{1}^{2} s_{2}^{2}\right)}
\end{aligned}
$$

The net (interim) payoff of publication is the sum of the right-hand sides of $\sqrt{19},(21)$, and $(22)$, minus $c$. To get the form stated in the proposition, we add up the coefficients on $\left(X_{1}-\mu_{0}\right)^{2}$ in (19) and (21):

$$
\alpha \frac{\sigma_{0}^{4}}{\left(\sigma_{0}^{2}+s_{1}^{2}\right)^{2}}+(1-\alpha)\left(\frac{s_{2}^{2} \sigma_{0}^{2}}{\left(\sigma_{0}^{2}+s_{1}^{2}\right)\left(\sigma_{0}^{2}+s_{2}^{2}\right)}\right)^{2}=\frac{\sigma_{0}^{4}\left(s_{2}^{4}+2 \alpha \sigma_{0}^{2} s_{2}^{2}+\alpha \sigma_{0}^{4}\right)}{\left(\sigma_{0}^{2}+S_{1}^{2}\right)^{2}\left(\sigma_{0}^{2}+s_{2}^{2}\right)^{2}} .
$$




\section{Proof of Corollary 6 .}

1. This result is immediate.

2. The derivative of the benefit with respect to $\sigma_{0}$ evaluates to

$$
(1-\alpha) \frac{2 s_{2}^{4} \sigma_{0}^{7}\left(S_{1}^{4} \sigma_{0}^{4}+2 s_{2}^{4}\left(S_{1}^{2}+\sigma_{0}^{2}\right)\left(2 S_{1}^{2}+\sigma_{0}^{2}\right)+s_{2}^{2}\left(5 S_{1}^{4} \sigma_{0}^{2}+4 S_{1}^{2} \sigma_{0}^{4}\right)\right)}{\left(s_{2}^{2}+\sigma_{0}^{2}\right)^{3}\left(S_{1}^{2}+\sigma_{0}^{2}\right)^{2}\left(S_{1}^{2} \sigma_{0}^{2}+s_{2}^{2} S_{1}^{2}+s_{2}^{2} \sigma_{0}^{2}\right)^{2}}
$$

which is positive. As $\sigma_{0} \rightarrow 0$, the numerator goes to 0 while the denominator goes to a positive limit.

3. The derivative of the benefit with respect to $S_{1}$ evaluates to

$$
-(1-\alpha) \frac{2 s_{2}^{4} S_{1} \sigma_{0}^{8}\left(2 S_{1}^{2} \sigma_{0}^{2}+\sigma_{0}^{4}+2 s_{2}^{2} S_{1}^{2}+2 s_{2}^{2} \sigma_{0}^{2}\right)}{\left(s_{2}^{2}+\sigma_{0}^{2}\right)^{2}\left(S_{1}^{2}+\sigma_{0}^{2}\right)^{2}\left(S_{1}^{2} \sigma_{0}^{2}+s_{2}^{2} S_{1}^{2}+s_{2}^{2} \sigma_{0}^{2}\right)^{2}}
$$

which is negative. As $S_{1} \rightarrow \infty$, the numerator is constant while the denominator goes to infinity.

4. The derivative of the benefit with respect to $s_{2}$ evaluates to

$$
(1-\alpha) \frac{2 \sigma_{0}^{8} s_{2}^{3}\left(-s_{2}^{4}\left(S_{1}^{2}+\sigma_{0}^{2}\right)+s_{2}^{2} \sigma_{0}^{2}\left(S_{1}^{2}+\sigma_{0}^{2}\right)+2 S_{1}^{2} \sigma_{0}^{4}\right)}{\left(s_{2}^{2}+\sigma_{0}^{2}\right)^{3}\left(S_{1}^{2}+\sigma_{0}^{2}\right)\left(S_{1}^{2} \sigma_{0}^{2}+s_{2}^{2} S_{1}^{2}+s_{2}^{2} \sigma_{0}^{2}\right)^{2}} .
$$

This has the sign of $-s_{2}^{4}\left(S_{1}^{2}+\sigma_{0}^{2}\right)+s_{2}^{2} \sigma_{0}^{2}\left(S_{1}^{2}+\sigma_{0}^{2}\right)+2 S_{1}^{2} \sigma_{0}^{4}$. This expression is a concave quadratic in $s_{2}^{2}$, which is positive at $s_{2}^{2}=0$ and maximized at $s_{2}^{2}=\frac{\sigma_{0}^{2}}{2}>0$. In particular, the derivative in $s_{2}$ is positive and then negative.

As $s_{2} \rightarrow 0$, the numerator goes to zero while the denominator goes to a positive limit. As $s_{2} \rightarrow \infty$, the numerator increases at a rate of $s_{2}^{4}$ while the denominator increases at a rate of $s_{2}^{6}$, so the ratio goes to 0 .

Proof of Proposition 9. Write mean beliefs at the first period when a study $\left(X_{1}, S_{1}\right)$ is published or not by $\mu_{1}^{\left(X_{1}, S_{1}\right)}$ and $\mu_{1}^{0}$, and in the second period conditional on $X_{2}$ by $\mu_{2}^{\left(X_{1}, S_{1}\right),\left(X_{2}\right)}$ and $\mu_{2}^{0,\left(X_{2}\right)}$. The gross interim benefit of publishing a study $\left(X_{1}, S_{1}\right)$ can be expressed as follows as the first-period action benefit plus the expected second-period action benefit:

$$
\alpha\left(\mu_{1}^{\left(X_{1}, S_{1}\right)}-\mu_{1}^{0}\right)^{2}+(1-\alpha) \mathbb{E}_{\theta \sim \pi_{1}^{\left(X_{1}, S_{1}\right)}, X_{2} \sim \mathcal{N}\left(\theta, s_{2}^{2}\right)}\left[\left(\mu_{2}^{\left(X_{1}, S_{1}\right),\left(X_{2}\right)}-\mu_{2}^{0,\left(X_{2}\right)}\right)^{2}\right]
$$

The first term, the first-period action benefit, is nonnegative (and strictly positive when the means $\mu_{1}^{\left(X_{1}, S_{1}\right)}$ and $\mu_{1}^{0}$ differ). So it suffices to show that when $\pi_{1}^{\left(X_{1}, S_{1}\right)} \neq \pi_{1}^{0}$, the second term, the expected second-period action benefit, is strictly positive. In turn, it suffices to show that when $\pi_{1}^{\left(X_{1}, S_{1}\right)} \neq \pi_{1}^{0}$, there exists $X_{2}$ for which $\mu_{2}^{\left(X_{1}, S_{1}\right),\left(X_{2}\right)} \neq \mu_{2}^{0,\left(X_{2}\right)}$. (The second-period action benefit is nonnegative and is continuous in $X_{2}$, and $X_{2}$ has full support given any first-period interim belief $\pi_{1}^{\left(X_{1}, S_{1}\right)}$. So if the second-period action benefit is strictly positive at some $X_{2}$, then it is strictly positive in expectation.) The claim thus follows if we can show that, if $\mu_{2}^{\left(X_{1}, S_{1}\right),\left(X_{2}\right)}=\mu_{2}^{0,\left(X_{2}\right)}$ holds for all $X_{2}$, then $\pi_{1}^{\left(X_{1}, S_{1}\right)}=\pi_{1}^{0}$.

Without loss of generality, normalize $s_{2}=1$, so that $X_{2} \sim \mathcal{N}(\theta, 1)$. Define $m(x ; \pi)=\mathbb{E}_{\theta \sim \pi}\left[\theta \mid X_{2}=\right.$ $x]$ as the posterior mean of $\theta$ under $\pi$ when $X_{2}=x$. We seek to show that if $m(x ; \pi)=m(x ; \tilde{\pi})$ for all $x \in \mathbb{R}$, then $\pi=\tilde{\pi}$. 
Taking $\varphi$ to be the PDF of the standard normal, define $\pi * \varphi$ to be the marginal density of $X_{2}$ given $\theta \sim \pi$, which always exists:

$$
(\pi * \varphi)(x)=\int_{\mathbb{R}} \varphi(x-\theta) d \pi(\theta) .
$$

It then holds that

$$
\begin{aligned}
\frac{\partial \log ((\pi * \varphi)(x))}{\partial x} & =\frac{1}{(\pi * \varphi)(x)} \frac{\partial(\pi * \varphi)(x)}{\partial x}=\frac{\int_{\mathbb{R}} \varphi^{\prime}(x-\theta) d \pi(\theta)}{\int_{\mathbb{R}} \varphi(x-\theta) d \pi(\theta)}=\frac{\int_{\mathbb{R}}(\theta-x) \varphi(x-\theta) d \pi(\theta)}{\int_{\mathbb{R}} \varphi(x-\theta) d \pi(\theta)} \\
& =\mathbb{E}_{\theta \sim \pi}\left[\theta \mid X_{2}=x\right]-x=m(x ; \pi)-x
\end{aligned}
$$

where the last inequality on the first line follows from the identity $\varphi^{\prime}(x)=-x \varphi(x)$. Integrating the left- and right-hand sides yields

$$
(\pi * \varphi)(x)=C \cdot \exp \left(\int_{0}^{x}(m(x ; \pi)-x) d x\right)
$$

for a constant of integration $C$ pinned down by the fact that $\pi * \varphi$ integrates to 1 . The same formula holds for $\tilde{\pi} * \varphi$, replacing $\pi$ by $\tilde{\pi}$ on the right-hand side. We can therefore conclude that if $m(x ; \pi)=m(x ; \tilde{\pi})$ for all $x$, then $(\pi * \varphi)(x)=(\tilde{\pi} * \varphi)(x)$ for all $x$ as well.

So, suppose that $m(x, \pi)=m(x, \tilde{\pi})$ for all $x \in \mathbb{R}$, and hence that $(\pi * \varphi)(x)=(\tilde{\pi} * \varphi)(x)$. For any distribution $\pi$ of $\theta$, denote its characteristic function (Fourier transform) by $\psi_{\pi}(t)=E_{\theta \sim \pi}[\exp (i t \theta)]$. The fact that $\pi * \varphi=\tilde{\pi} * \varphi$ implies

$$
\psi_{\pi}(t) \cdot \exp \left(-t^{2} / 2\right)=\psi_{\tilde{\pi}}(t) \cdot \exp \left(-t^{2} / 2\right)
$$

for all $t$, where $\exp \left(-t^{2} / 2\right)$ is the characteristic function of the standard normal distribution. This holds because the Fourier transform maps convolutions of random variables into products of their characteristic functions. It immediately follows that $\psi_{\pi}(\cdot)=\psi_{\tilde{\pi}}(\cdot)$, since $\exp \left(-t^{2} / 2\right)$ is different from 0 for all $t$, so that the characteristic function of $\pi$ is equal to the characteristic function of $\tilde{\pi}$. It follows that $\pi$ and $\tilde{\pi}$ are equal, by Lemma 2.15 in Van der Vaart (2000).

Proof of Proposition 10. We first state a lemma that does not depend on Assumption 1.

Lemma 4. In searching for an incentive-optimal publication rule, it is without loss of generality to restrict to rules $p(X, S)$ satisfying

$$
p(X, S)=\left\{\begin{array}{c}
1 \quad \text { if } S=\bar{s} \text { and } \Delta\left(\pi_{1}^{(X, S)}, a^{*}\left(\pi_{0}\right)\right)>c-\lambda, \\
\quad \text { or if } S<\bar{s} \text { and } \Delta\left(\pi_{1}^{(X, S)}, a^{*}\left(\pi_{0}\right)\right) \geq c \\
0 \quad \text { if } S>\bar{s}, \\
\quad \text { or if } S=\bar{s} \text { and } \Delta\left(\pi_{1}^{(X, S)}, a^{*}\left(\pi_{0}\right)\right)<c-\lambda \\
\quad \text { or if } S<\bar{s} \text { and } \Delta\left(\pi_{1}^{(X, S)}, a^{*}\left(\pi_{0}\right)\right)>c
\end{array}\right.
$$

for some $\bar{s} \in(0, \infty)$ and $\lambda$ in $\mathbb{R} \cup\{-\infty, \infty\}$ in which the researcher chooses $S=\bar{s}$ if she conducts a study.

It remains only to show that in the incentive-optimal contract of the form in Lemma 4 the researcher chooses to conduct a study; that $\bar{s} \leq s^{\text {int }}$; and that $\lambda \geq 0$.

The facts that the researcher conducts a study and that $\bar{s} \leq s^{\text {int }}$ both follow from Assumption 1 .

First, Assumption 1 guarantees that the journal prefers to follow the interim-optimal rule - at which the researcher conducts a study with $S=s^{\text {int }}$, and the journal only publishes studies with a nonnegative interim net benefit - than any rule that publishes nothing at all. (In the model without 
incentives in which $q=1$ and $S$ is deterministically equal to $s^{\text {int }}$, publishing no studies is feasible, but the interim-optimal rule is preferred.) So the incentive-optimal rule will induce the researcher to conduct a study, meaning that the researcher must be choosing $S=\bar{s}$.

Second, fix any publication rule of the form in Lemma 4 with $\bar{s}=s^{h}$ and $\lambda=\lambda^{h}$, for $s^{h}>s^{\text {int }}$. We claim that the publication rule of the same form with $\bar{s}=s^{\text {int }}$ and $\lambda=0$ weakly improves payoffs. To see why this claim holds, note that the publication rule with $\bar{s}=s^{h}$ and $\lambda=\lambda^{h}$ would be weakly improved upon by one with $\bar{s}=s^{\text {int }}$ and $\lambda=0$, supposing researcher participation. Recall that normal signals are Blackwell ordered by their standard errors: at standard error $S=s^{\text {int }}$, the findings $X$ can be garbled into something informationally equivalent to findings from $S=s^{h}$. So some stochastic publication rule at $S=s^{\text {int }}$, combined with a garbling of these signals to the public, replicates the distribution of outcomes (probability of publication at each state, and joint distribution over public actions and states conditional on publication) that occur when a study arrives with $S=s^{h}$ and is published under the publication rule given by $\bar{s}=s^{h}$ and $\lambda=\lambda^{h}$. But the journal's payoff given a study with $S=s^{\text {int }}$ are improved by removing the garbling to the public. Payoffs are further improved by publishing under the interim-optimal publication rule at $S=s^{\text {int }}$, which is exactly that given by a rule of the form in Lemma 4 with $\bar{s}=s^{\text {int }}$ and $\lambda=0$. Finally, by Assumption 1, the publication rule with $\bar{s}=s^{\text {int }}$ and $\lambda=0$ does indeed get researcher to conduct a study, since the interim outcome satisfies the researcher's participation constraint.

The final step is to show that $\lambda \geq 0$. This is because, for any publication rule of the form of Lemma 4 increasing $\lambda$ increases the publication probability at $S=\bar{s}$. Hence, it makes the researcher better off if she chooses $S=\bar{s}$ and slackens her incentive constraints. Moreover, starting from $\lambda<0$, increasing $\lambda$ to 0 improves the journal's payoff, since again $\lambda=0$ is interim optimal and hence optimal conditional on a study being submitted at $S=\bar{s}$.

Proof of Lemma 4. Take an arbitrary publication rule $\tilde{p}$. We will show that it can be replaced by a rule $p$ of the desired form that weakly increases the journal's payoff.

First suppose that $\tilde{p}$ does not induce the researcher to conduct a study. Then define some $p$ of the form in the statement of the Lemma by setting $\bar{s}$ arbitrarily and setting $\lambda=0$. If the publication rule $p$ induces the researcher not to participate, then the journal's payoffs are unchanged from $\tilde{p}$. If the rule $p$ induces the researcher to conduct a study with standard error $S=s$, then the journal's payoffs are weakly higher than before, since under $p$ the journal never publishes studies that give negative net interim payoff.

So, for the rest of the proof, assume that $\tilde{p}$ does in fact induce the researcher to conduct a study with $S$ equal to some level $\bar{s}$. We show that there exists $\lambda$ such that we can replace $\tilde{p}$ with a publication rule $p$ satisfying the following properties and weakly improve the journal's payoff:

1. At $s>\bar{s}, p(x, s)=0$ :

Let $p(x, s)=\tilde{p}(x, s)$ at $s \leq \bar{s}$ and 0 at $x>\bar{s}$. The publication rule $p$ gives the researcher the same payoff from choosing $S=\bar{s}$ and weakly reduces her payoff from choosing other values of $S$, and so under $p$ the researcher's behavior is unchanged. She continues to conduct a study with $S=\bar{s}$ and the journal's payoff given the choice of $S=\bar{s}$ is also unchanged.

2. At $s=\bar{s}, p(X, \bar{s})=1$ if $\Delta\left(\pi_{1}^{(X, \bar{s})}, a^{*}\left(\pi_{0}\right)\right)>c-\lambda$, and $p(X, \bar{s})=0$ if $\Delta\left(\pi_{1}^{(X, \bar{s})}, a^{*}\left(\pi_{0}\right)\right)<c-\lambda$ : Let $p(x, s)=\tilde{p}(x, s)$ at all $s \neq \bar{s}$. Denote the probability of publication under $\tilde{p}$ at $S=\bar{s}$, given by $\mathbb{E}[\tilde{p}(X, S) \mid S=\bar{s}]$, by $y \in[0,1]$. If $y=0$ then $\tilde{p}$ is equivalent to a publication rule $p$ of the appropriate form with $\lambda=\infty$. If $y=1$ then $\tilde{p}$ is equivalent to a publication rule $p$ of the appropriate form with $\lambda=-\infty$.

For interior $y$, define $p(\cdot, \bar{s})$ so as to maximize the journal's payoff subject to accepting a share $y$ of papers at this standard error. To do so, first set $\lambda \in \mathbb{R}$ as the supremum over values of $l$ such that $P\left(\Delta\left(\pi_{1}^{(X, \bar{s})}, a^{*}\left(\pi_{0}\right)\right)>c-l \mid S=\bar{s}\right] \leq y$. Next, let $p(x, \bar{s})=0$ if $\Delta\left(\pi_{1}^{(x, \bar{s})}, a^{*}\left(\pi_{0}\right)\right)<c-\lambda$ and let $p(x, \bar{s})=1$ if $\Delta\left(\pi_{1}^{(x, \bar{s})}, a^{*}\left(\pi_{0}\right)\right)>c-\lambda$. Finally, if $\Delta\left(\pi_{1}^{(x, \bar{s})}, a^{*}\left(\pi_{0}\right)\right)=c-\lambda$, set $p(x, \bar{s})$ 
such that the publication probability at $S=\bar{s}, \mathbb{E}[p(X, S) \mid S=\bar{s}]$, is equal to $y$. (This last step is only relevant if $\Delta\left(\pi_{1}^{(X, \bar{s})}, a^{*}\left(\pi_{0}\right)\right)=c-\lambda$ with positive probability at $S=\bar{s}$.)

The publication rules $p$ and $\tilde{p}$ publish with probability as each other conditional on any choice $S$ by the researcher. Hence, the researcher continues to be willing to pick $S=\bar{s}$. Moreover, given the constraint of publishing with probability $y$ at $S=\bar{s}$, the journal's expected payoff given a researcher choice of $S=\bar{s}$ is maximized by $p$. Hence, the journal weakly prefers $p$ to $\tilde{p}$ if the researcher is to choose $S=\bar{s}$.

3. At $s<\bar{s}, p(x, s)=1$ if $\Delta\left(\pi_{1}^{(x, s)}, a^{*}\left(\pi_{0}\right)\right) \geq c$ and $p(x, s)=0$ if $\Delta\left(\pi_{1}^{(x, s)}, a^{*}\left(\pi_{0}\right)\right)<c$ :

Let $p(x, s)=\tilde{p}(x, s)$ at $s \geq \bar{s}$; at $s<\bar{s}$, let $p(x, s)=1$ if $\Delta\left(\pi_{1}^{(x, s)}, a^{*}\left(\pi_{0}\right)\right) \geq c$ and $p(x, s)=0$ if $\Delta\left(\pi_{1}^{(x, s)}, a^{*}\left(\pi_{0}\right)\right)<c$.

Under publication rule $p$, the researcher will either continue to choose $S=\bar{s}$ or will switch to $s^{\prime}<\bar{s}$. If the researcher continues to choose $S=\bar{s}$, then the journal's payoffs are as before. If the researcher now chooses $s^{\prime}<\bar{s}$, we claim that the journal must be weakly better off. (This argument exactly follows an argument in the proof of Proposition 10.) To show the claim, recall that normal signals are Blackwell ordered by their standard errors: at standard error $S=s^{\prime}$, the finding $X$ can be garbled into something informationally equivalent to a finding from $S=\bar{s}$. So some stochastic publication rule at $S=s^{\prime}$, combined with a garbling of these signals to the public, replicates the distribution of outcomes (probability of publication at each state, and joint distribution over public actions and states conditional on publication) that occur when a study arrives with $S=\bar{s}$ and is published under the publication rule given by $p(X, \bar{s})$. But the journal's payoffs given a study that has been published with $S=s^{\prime}$ are improved by removing the garbling to the public. Payoffs are further improved by publishing under the interim-optimal publication rule at $S=s^{\prime}$, which is exactly that under $p$.

The only remaining item to prove is that it is without loss of generality to suppose that if the researcher chooses to conduct a study, she chooses $S=\bar{s}$; applying step 3 above could possibly have changed the researcher's choice of $S$ to something below $\bar{s}$. However, iterating step 1 (with $\bar{s}$ redefined to the new choice of $S$ ) recovers a publication rule of the appropriate form in which the researcher does choose $S=\bar{s}$.

Proof of Proposition 11. Recall that under normal priors and normal signals, the variance of $\pi_{1}^{(X, S)}$ is independent of $X$. So fix $S=s$, and without loss of generality normalize the variance of $\pi_{1}^{(X, s)}$ to 1 . Then given $X=x$ and $\theta \sim \pi_{1}^{(x, s)}$, the distribution of a random variable $Y=(x-\theta)^{2}$ is a noncentral chi-squared distribution with noncentrality parameter $\lambda$ (equal to $\left(x-\mathbb{E}_{\theta \sim \pi_{1}^{(x, s)}}[\theta]\right)^{2}$ ) that increases in $\left(x-\mu_{0}\right)^{2}$. The variable $Y$ has CDF over realizations $y$ given by $1-Q_{1 / 2}(\sqrt{\lambda}, \sqrt{y})$ for $Q$ the Marcum $Q$-function $2{ }^{26}$ By Sun et al. (2010) Theorem 1(a), $Q_{1 / 2}(\sqrt{\lambda}, \sqrt{y})$ strictly increases in its first term $\sqrt{\lambda}$, implying that the distribution of $(x-\theta)^{2}$ under $\pi_{1}^{(x, s)}$ increases in the sense of FOSD as $\left(x-\mu_{0}\right)^{2}$ increases, and hence that $\mathbb{E}_{\theta \sim \pi_{1}^{(x, s)}}\left[\delta\left((x-\theta)^{2}\right)\right]$ increases in $\left(x-\mu_{0}\right)^{2}$. A study $(X, S)=(x, s)$ is published if and only if $\mathbb{E}_{\theta \sim \pi_{1}^{(x, s)}}\left[\delta\left((x-\theta)^{2}\right)\right] \leq b$, so at standard error $S=s$ studies are published only if $\left(X-\mu_{0}\right)^{2}$ is sufficiently small.

\footnotetext{
${ }^{26}$ See Wikipedia for details: https://en.wikipedia.org/wiki/Noncentral_chi-squared_distribution
} 